\title{
Winding angle distribution for planar random walk, polymer ring entangled with an obstacle, and all that: Spitzer-Edwards-Prager-Frisch model revisited
}

\author{
A. Grosberg ${ }^{1,2}$ and H. Frisch ${ }^{3,4}$ \\ ${ }^{1}$ Department of Physics, University of Minnesota, Minneapolis, MN 55455, USA \\ ${ }^{2}$ Institute of Biochemical Physics, Russian Academy of Sciences, Moscow 117977, Russia \\ ${ }^{3}$ Department of Chemistry, State University of New York at Albany, Albany, NY 12222, USA \\ ${ }^{4}$ Physics Department, Stellenbosch University, Matieland, 7602 South Africa
}

(Dated: November 21, 2018)

\begin{abstract}
Using a general Green function formulation, we re-derive, both, (i) Spitzer and his followers results for the winding angle distribution of the planar Brownian motion, and (ii) Edwards-Prager-Frisch results on the statistical mechanics of a ring polymer entangled with a straight bar. In the statistical mechanics part, we consider both cases of quenched and annealed topology. Among new results, we compute exactly the (expectation value of) the surface area of the locus of points such that each of them has linking number $n$ with a given closed random walk trajectory (= ring polymer). We also consider the generalizations of the problem for the finite diameter (disc-like) obstacle and winding within a cavity.

PACS numbers: 61.41.+e, 36.20.Ey, 87.15.Cc
\end{abstract}

\section{INTRODUCTION}

In 1958, Spitzer [1] established the following result. Consider the two-dimensional random walk starting at a point other than $\mathcal{O}$, and let $\theta(t)$ be the total continuous angle wound by the walker around $\mathcal{O}$ up to time $t$ (see figure 1a). The Spitzer law says that the quantity $\theta(t) / \ln t$ at large enough $t$ is Lorenz (or Cauchy) distributed:

$$
W(\theta)=\frac{1}{\pi} \frac{1}{1+x^{2}} ; x=\frac{2 \theta(t)}{\ln t} .
$$

With a remarkable delay of about 25 years, a large group of followers studied this law in depth $2,3,3,4,5,6,6,7,8$, 9, 10, 11, 12, 13. The central finding of these studies attributes the divergent moments of the Spitzer distribution (1), e.g. $\left\langle\theta^{2}\right\rangle$, to the small scale properties of the regular random walk trajectories. Simply speaking, infinitely large winding is accumulated while the trajectory is wandering infinitely close to the obstacle $\mathcal{O}$. Accordingly, this pathology of divergent moments is removed by incorporating any kind of "granularity," or short length scale cut-off, in the model. Such modification of the model can be achieved in quite a few ways. One way is to consider the random walk on the lattice instead of the continuous space [9]; another way is to look at the winding around a finite obstacle, say, a disc of some radius $b[2,[4,[5]$; one more possibility is to examine a broken line of straight segments of finite length $b$ each instead of standard Wiener-measured random walk; yet another way is to consider a worm-like smooth curve with an effective segment $b$ (that is, the curve which adopts smoothly curved shapes $\mathbf{r}(s)$ with the weight proportional to $\exp \left[-(b / 2) \int \ddot{\mathbf{r}}^{2} d s\right]$, where $s$ is the arc length). In all of these cases, winding is characterized by the nonpathological distribution

$$
W(\theta)=\frac{\pi}{4 \cosh ^{2}(\pi x / 2)} ; x=\frac{2 \theta(t)}{\ln t} .
$$

A similar distribution is also characteristic for the winding of the self-avoiding walk [3, 6, 7]; self-avoidance, in this case, is just another way to suppress infinite winding at infinitely small length scale. Mathematically, it turns out that the winding angle distribution is in fact an example of a broad class of limiting laws for the twodimensional random walk 4,8$]$.

Many studies of winding angle distribution 2, 3, 3, 5, 6, 7, 10, 12, 13 claim that entanglement of long polymer filaments is (one of) their motivation(s). Indeed, the relation to polymer physics does exist. It was found in 1967, almost a decade after Spitzer [1], by Edwards [14] and, independently, by Prager and Frisch [15] (see also an influential review 16]). These authors came up with the model of a polymer chain wound around a straight bar and topologically entangled with this bar. Given the analogy of a polymer chain conformation with the random walk trajectory, the Edwards-Prager-Frisch model is essentially the same as that examined by Spitzer [1]. Neither of the works 14, 15, 16] makes a reference to [1]. Most likely, mathematical work 1] was not known to physicists at the time, but even apart from that, authors of the works 14, 15, 16] did not examine winding angle distribution for the random walk with open ends, their goal was obviously to compute quantities similar to those of physical interest for real polymers. Unfortunately, no explicit formula was obtained in the works [14, 15, 16] comparable in simplicity to Eq. (11).

To our surprise, we found that this fairly old area lacks both unity and clarity. The studies of winding angle distribution [2, 3, 4, 5, 6, 6, 8, 9, 10] contain no hint on the lessons of this exactly solvable model to polymer physics. Drossel and Kardar 11] as well as Samokhin 12, 13. brought the subject to a new level of complexity, they examined winding angle distribution for the random walks in a disordered medium. Drossel and Kardar 11] also provided simple derivation of the results (12) and applied it to many physical situations involving directed polymers, but all that yields little insight into the topological 
properties of ring polymers. And we are unaware of any followers of [14, 15, 16] who took advantage of the more recent mathematical achievements [2, 4, 5, 6, 8, 9, 10]. Meanwhile, an exactly solvable model in general is useful if only it yields some insight(s). Upon a closer look and re-examination of the literature, we found that the model of winding can be made to meet this criteria, but it has not been done yet. Our plan in this paper is to reconsider the problem from a single common view point, including both winding angle distribution and some more physical aspects.

Our additional motivation arises from the fact that the study of topological constraints in polymers in the years and decades after the works [14, 15, 16] had been dominated by the phenomenological approaches based on the reptation theory [17, 18]. At the same time, a breakthrough in microscopic understanding of this subject has not been achieved, and, therefore, the need for exactly solvable models remains high. Moreover, apart from networks, there is now another large "consumer" for polymer topology, this is DNA physics. The DNA double helix is frequently found in a closed loop form, it forms knots of various kinds [19, 20], and there are special enzymes spending energy to simplify the entanglements 21].

One of the key aspects of polymer topology is that there are two types of questions one can ask, corresponding to annealed and quenched topological disorder, respectively [22]. The beauty of the winding model, which so far seems to remain underappreciated, is that it allows both types of questions:

- The typical annealed topology question is that about ring closure experiment and knot probabilities 25, 26, 27, 28, 29]: having a linear polymer with "sticky" ends, what is the probability to obtain a certain type of a knot upon first meeting of the two ends [19, 20]? A similar question for the winding model is this: what is the probability that a random walk on the plane links number $n$ (or winding angle $2 \pi n$ ) with an obstacle?

- The typical quenched topology question is about, e.g., the size or other properties of a polymer having a given fixed topology (e.g, knot type) [23, 24]; this is necessary, e.g., to understand the diffusion of knotted DNA in solution or in a gel. A s imilar question for the winding model is this: given a polymer with fixed linking number $n$, what is the (root-mean-squared) average distance of an arbitrary point on the trajectory from $\mathcal{O}$ ?

To conclude the introduction, we should also mention that the shortcomings of the Edwards-Prager-Frisch model are well understood 31]. Basically, this model assumes that entanglements algebraically commute with each other, while the real physical situation is nonAbelian.

This paper is organized as follows. In section III we discuss the Green function formulation of the problem and derive basic equations for all models - winding
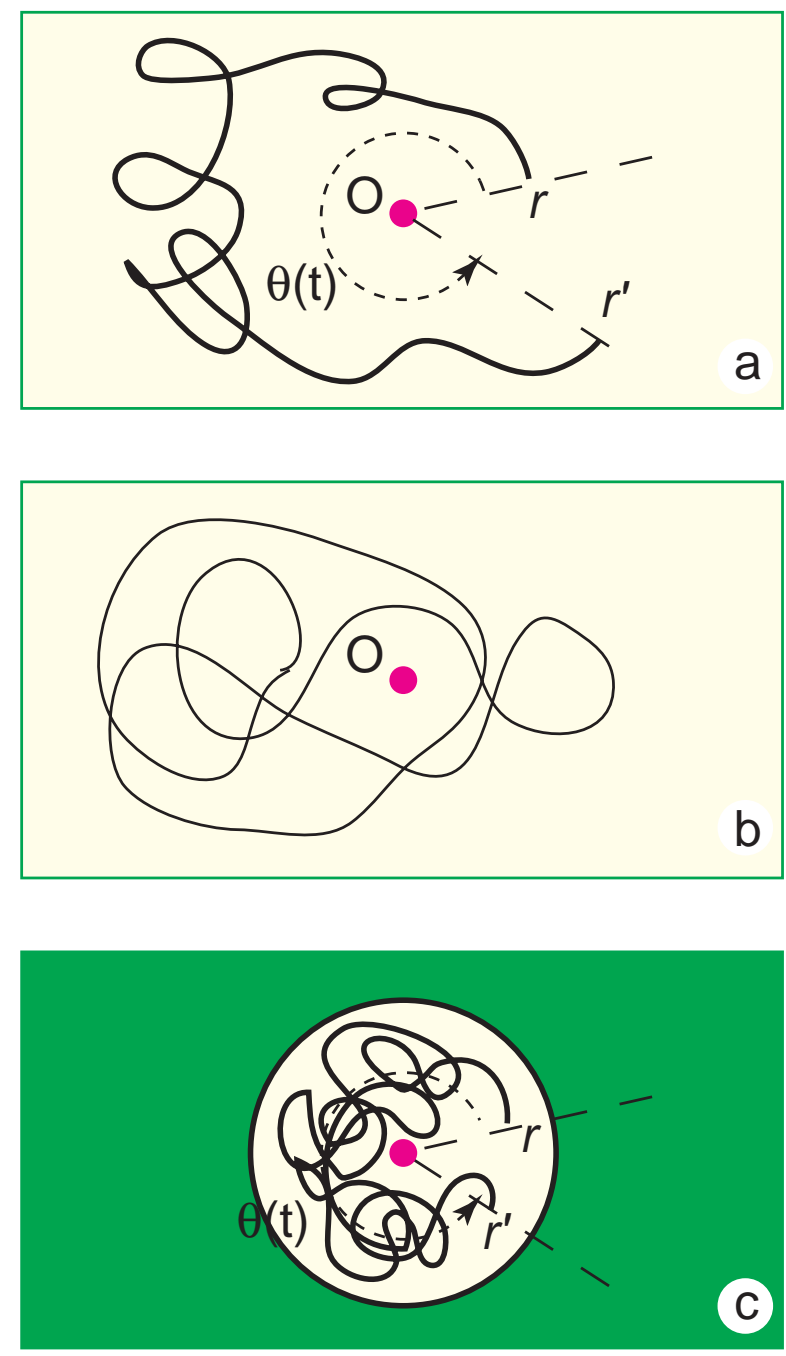

FIG. 1: Schematic representattion of the model. (a) Random walk winding around an obstacle $\mathcal{O}$. This obstacle might be just a point, or it might be a disc of a finite radius $b$. (b) Closed polymer winding around an obstacle. Mathematically, this is similar to (a), except both ends are kept together. (c) Similar to (a), except the trajectory cannot leave a "cavity" of some radius $B$.

around the point, around the disc, or inside the cavity. In section [V] we show how to re-derive and generalize the results (11) and (2). In section V we consider the closed loop polymer, which is the random walk with connected ends. In section VI we make a few final comments.

Our work is heavy on calculations, even though some of the less important ones are relegated into Appendices. As readers, we don't like such heavy papers. This is why we start from section \which provides an overview of major steps and the results for those readers not interested in details. 


\section{BIRD'S EYE VIEW OF THE RESULTS: FOR THE LAZY READER WHO DOES NOT WANT TO DWELL ON THE CALCULATIONS}

If you, our reader, do not want to follow our calculations, this section offers a tour of the results for you.

To begin with, section 11 contains no results: it describes the standard diffusion equation and bilinear expansion of its Green function over the appropriate set of Bessel functions. Here, for a "tourist," all that is necessary to know is the notation $a^{2} / 4$ adopted for the diffusion coefficient, which means that the root-mean-square distance traveled by a walker during the time $t$ is equal to $a \sqrt{t}$.

Formula (14) in section IV A is our first small result, it is a very mild generalization of the Spitzer formula (II) which takes explicit account of the distances $r$ and $r^{\prime}$ of a polymer (or random walk trajectory) ends from the origin (or obstacle) $\mathcal{O}$. Formula (14) gives the probability distribution of winding angle $\theta$ for the random walk of length $t$ with $r$ and $r^{\prime}$ fixed. Like the Spitzer law, it has diverging moments, such as $\left\langle\theta^{2}\right\rangle$.

The very cumbersome formula (23) gives a similar result for the winding around a disc of a finite radius $b$. It generalizes formula (2) by keeping explicit track of positions $r$ and $r^{\prime}$ of both ends. Just like (2), it decays exponentially and yields finite values for all moments, e.g., $\left\langle\theta^{2}\right\rangle$. One utility of this result is the analysis of cross-over between winding around a point with infinite $\left\langle\theta^{2}\right\rangle$ and winding around a disc with finite $\left\langle\theta^{2}\right\rangle$. As we show in Section IVC when the disc size $b$ goes to zero, there opens a wide range of times $t$ (see Eq. (26)) where the probability behaves as $\sim 1 / \theta^{2}$ up to $\theta$ about $\ln (a / b)$, and only at larger $\theta$ exponential decay takes over; therefore, when we say that $\left\langle\theta^{2}\right\rangle$ diverges, this really means $\left\langle\theta^{2}\right\rangle \sim(\ln (a / b))^{2}$ at small $b$.

In Section $\nabla$ we go closer to the polymer view on the subject. For this, we consider that the two ends of the random walk are glued together, so that $r=r^{\prime}$ and $\theta=2 \pi n$, where (positive or negative) integer $n$ is the linking number, the number of turns the polymer ring makes around the obstacle. Figure 2 depicts the statistical weight of the polymer conformations with the linking number $n$ as a function of $r^{2} / t$. Qualitatively, this exhibits a behavior similar to that of the knotting probability as a function of chain length, because all cases with $n \neq 0$ (similar to non-trivial knots) reach maximal weight at some intermediate values of $r$ and/or $t$.

Sections $\mathrm{VB}$ and $\mathrm{VC}$ present our most original and most interesting findings. In particular, we consider the following question. Given the closed random walk trajectory of the length $t$, we consider $\Sigma_{n}$ - the locus of points around which the trajectory makes exactly $n$ turns. Then, what is the area of $\Sigma_{n}$ ? We denote by $\sigma_{n}(t)$ the average (over the random walk trajectories) of this area, then formula (44) provides the exact answer to this question. The essence is that $\sigma_{n}(t)$ decreases very slowly with $n$, only as $1 / n^{2}$. Of course, ideologically this is similar to the slow decay of the Spitzer distribution (11). Another look at the same result is to think about a virial coefficient of a polymer ring with a long straight bar. Their interaction is topological in nature [25], and the virial coefficient can be understood as the surface excluded for a polymer ring by the presence of the obstacle if the ring is not entangled with the obstacle. This virial coefficient is the sum of all $\sigma_{n}(t)$ with $n \neq 0$, and it is exactly equal to $\pi t a^{2} / 12$.

Note that the former view of $\sigma_{n}(t)$ corresponds to the question about annealed topological disorder, as it relates $\sigma_{n}(t)$ to the probability of getting the topological state $n$. By contrast, the latter view on the same quantities $\sigma_{n}(t)$ corresponds closer to the idea of quenched topological disorder, as it reflects on the physical property of the polymer with given $n$. Another such physical quantity is the distance between the obstacle and an arbitrary point on the polymer. The exact expression for the rootmean-square of such distance is given by formula (48). The interesting aspect of this result is that this distance remains of the order of $a \sqrt{t}$ and only quite modestly depends on $n$, changing from approximately $0.496 a \sqrt{t}$ when $n=1$ to $0.408 a \sqrt{t}$ when $n \rightarrow \infty$. The fact that this distance decreases with growing "topological complexity" $n$ is not surprising, but the fact that it changes only slightly is interesting. One could have thought that the polymer would consist of $n$ roughly similar loops, leading to the typical size of $a \sqrt{t / n}$. Our result, therefore, suggests that even at very large $n$ there remains one big loop, with the length of order $t$, while all other loops are tight and small. This is reminiscent of knot tightening recently discussed by Kardar and his co-workers 35].

In section VD we make a brief comment on the elastic forces developing in the polymer ring either pushed too close to the obstacle or pulled too far away from it.

Finally, in the section $\mathrm{VE}$ we consider polymer ring entangled with a finite size obstacle, and show that in this case the distribution over the linking number $n$ decays exponentially at large $n$, and the characteristic $n$ is about $\ln \left(t a^{2} / b^{2}\right)$.

\section{GREEN FUNCTION FORMULATION}

\section{A. Point-like obstacle}

Consider a Gaussian polymer in $2 D$ or, equivalently, a random walk in $2 D$. Suppose first that the obstacle is point-like, positioned at $\mathcal{O}$, the coordinate center. The statistics of trajectories is fully described by the Green function, $G\left(\begin{array}{c|c}\vec{r}^{\prime} & \vec{r} \\ 0 & t\end{array}\right)$, which is the partition function (or statistical weight) of the chain having the monomer 0 at $\vec{r}^{\prime}$ and monomer $t$ at $\vec{r}$. The Green function satisfies the diffusion equation

$$
\partial_{t} G\left(\begin{array}{c|c}
\vec{r}^{\prime} & \vec{r} \\
0 & t
\end{array}\right)=\frac{a^{2}}{4} \Delta G\left(\begin{array}{c|c}
\vec{r}^{\prime} & \vec{r} \\
0 & t
\end{array}\right)+\delta(t) \delta\left(\vec{r}-\vec{r}^{\prime}\right),
$$


where the notations are standard: $\Delta$ is the Laplace operator acting on $\vec{r}, a$ is the monomer size, $t$ is polymer length ("time"). The notation $a^{2} / 4$ adopted here for the diffusion coefficient, which is in fact $a^{2} / 2 d, d$ being space dimension, is convenient because root-mean-squared endto-end distance of the trajectory with no obstacles equals exactly $a \sqrt{t}$. The Green function can be written in terms of the bi-linear expansion over the corresponding eigenfunctions. Because our goal is to address the obstacle at $\mathcal{O}$, we choose eigenfunctions with cylindrical symmetry. The ones with no singularity at $\mathcal{O}$ read $J_{\mu}(\kappa r) e^{ \pm \imath \mu \theta}$, where $J_{\mu}(x)$ is Bessel function of the first kind, $r$ and $\theta$ are polar coordinates corresponding to $\vec{r}$, and $-\kappa^{2}$ is the corresponding eigenvalue. Accordingly, we write

$$
\begin{aligned}
G\left(\begin{array}{c|c}
r^{\prime}, 0 & r, \theta \\
0 & t
\end{array}\right) & =\frac{1}{2 \pi} \int_{0}^{\infty} \int_{0}^{\infty} e^{-a^{2} \kappa^{2} t / 4} \cos (\mu \theta) \times \\
& \times J_{\mu}(\kappa r) J_{\mu}\left(\kappa r^{\prime}\right) \kappa d \kappa d \mu .
\end{aligned}
$$

It is worth noting explicitly that only positive $\mu>0$ contribute to this expansion, because $J_{\mu}(x)$ with negative index $\mu$ is singular at small $x$.

In most cases in mathematical physics, the angular $\theta$ dependence is $2 \pi$-periodic, meaning that $\theta$ and $\theta \pm 2 \pi n$ label one and the same place on the plane. This is not the case for the problem at hand. Indeed, $G_{t}\left(r, 0 \mid r^{\prime}, \theta\right)$ is the statistical weight of trajectories (polymer conformations) that start at a point some distance $r$ away from the origin $\mathcal{O}$ and arrive after "time" $t$ at another point some $r^{\prime}$ from $\mathcal{O}$, where it is assumed that by the time $t$ the trajectory has accumulated winding angle $\theta$ around $\mathcal{O}$. Accordingly, for instance, $\theta=0$ means no turns around $\mathcal{O}$, while $\theta=$ $2 \pi$ means one turn counterclockwise, $\theta=-2 \pi$ is one turn clockwise, etc. In other words, we should treat our plane as a Riemann surface, in which case $\theta$ and $\theta \pm 2 \pi n$ correspond to different layers.

Most immediately, this means that not only integer, but all positive values of $\mu$ must be included in the bilinear expansion (4).

It turns out that integration over $\kappa$ can be explicitly performed; the derivation of the relevant so-called Weber integral [32] is provided in the Appendix A The result reads:

$$
\begin{aligned}
G\left(\begin{array}{c|c}
r^{\prime}, 0 & r, \theta \\
0 & t
\end{array}\right) & =\frac{2}{\pi a^{2} t} e^{-\left(r^{2}+r^{\prime 2}\right) / a^{2} t} \times \\
& \times \int_{0}^{\infty} \cos (\mu \theta) I_{\mu}\left(\frac{2 r r^{\prime}}{a^{2} t}\right) d \mu,
\end{aligned}
$$

where $I_{\mu}(x)$ is the modified Bessel function.

It is instructive to re-write the latter formula by introducing $R$ - the distance between $\vec{r}$ and $\vec{r}^{\prime}: \vec{R}=\vec{r}-\vec{r}^{\prime}$, or $R^{2}=r^{2}+r^{\prime 2}-2 r r^{\prime} \cos \theta$. We can write

$$
G\left(\begin{array}{c|c}
r^{\prime}, 0 & r, \theta \\
0 & t
\end{array}\right)=\left[\frac{1}{\pi a^{2} t} e^{-R^{2} / a^{2} t}\right] W\left(\theta, \frac{2 r r^{\prime}}{a^{2} t}\right),
$$

where

$$
W\left(\theta, \frac{2 r r^{\prime}}{a^{2} t}\right)=2 e^{-2 r r^{\prime} \cos \theta / a^{2} t} \times
$$

$$
\times \int_{0}^{\infty} \cos (\mu \theta) I_{\mu}\left(\frac{2 r r^{\prime}}{a^{2} t}\right) d \mu,
$$

The first factor in the Eq. (6) (in square brackets) is simply the Green function of an unrestricted polymer, or unrestricted random walk; in other words, it is the statistical weight of all conformations going from $\vec{r}$ to $\vec{r}$. Therefore, $W$ measures the fraction of trajectories with winding angle $\theta$ on the way.

Equations (517) were derived by Edwards [14].

\section{B. Finite size obstacle}

Consider now an obstacle having the shape of a disc with some finite radius $b$. Since the trajectory cannot make infinitely many turns around such obstacle, we expect that the probability distribution for the number of turns should be completely different for this case as compared to the point-like obstacle.

We use the same method as before. Eq. (31) still applies, but as regards bi-liner expansion, Eq. (4), we have now different set of eigenfunctions - the ones which satisfy the boundary condition of being equal to zero at $r=b$. This boundary condition removes all trajectories which cross the boundary, or, in other words, which enter the $r<b$ region. The eigenfunctions, corresponding to the eigenvalue $-\kappa^{2}$, can be written in the form $Z_{\mu}(\kappa r, \kappa b) e^{\imath \mu \theta}$, where (see Appendix B)

$$
Z_{\mu}(\kappa r, \kappa b)=\frac{-J_{\mu}(\kappa r) Y_{\mu}(\kappa b)+J_{\mu}(\kappa b) Y_{\mu}(\kappa r)}{\sqrt{J_{\mu}^{2}(\kappa b)+Y_{\mu}^{2}(\kappa b)}} .
$$

Here $Y_{\mu}(x)$ is Bessel function of the second kind (another frequently used notation for $Y_{\mu}(x)$ is $N_{\mu}(x)$; we adopt here the notation used in Mathematica 33|). A few notes about functions $Z$ are provided in the Appendix $B$ including the proof that the square root in the denominator makes them correctly normalized. Using $Z$, we write the Green function as a bi-linear expansion, like Eq. (4):

$$
\begin{aligned}
G\left(\begin{array}{c|c}
r^{\prime}, 0 & r, \theta \\
0 & t
\end{array}\right) & =\frac{1}{2 \pi} \int_{0}^{\infty} \int_{0}^{\infty} e^{-a^{2} \kappa^{2} t / 4} \cos (\mu \theta) \times \\
& \times Z_{\mu}(\kappa r, \kappa b) Z_{\mu}\left(\kappa r^{\prime}, \kappa b\right) \kappa d \kappa d \mu .(9)
\end{aligned}
$$

Unfortunately, no known analog exists of the Weber integral for the $Z$-functions, and so, unlike the $b=0$ case above, we were unable to find any way to simplify this by performing either of the two integrations.

Addressing the same problem of winding around the disk, Rudnick and $\mathrm{Hu}[5]$ have already found the expression for the Green function. Formula (9) looks surprisingly different from the known result [5]. In the Appendix C]we show explicitly that these two results are equivalent.

\section{Winding inside the cavity}

Yet another interesting model is shown in Fig. I(c). It is a random walk or linear polymer confined in a re- 
stricted volume, say, inside the disc of some radius $B$. Then, absorbing boundary conditions should be imposed on this boundary. Assuming for simplicity that the obstacle is located in the center of the confinement disc, we obtain that Eq. (4) holds, except integration over $\kappa$ at every $\mu$ must be replaced by the sum over the discrete spectrum of $\kappa_{n}(\mu)$ such that $J_{\mu}\left(\kappa_{n}(\mu) B\right)=0$. As usually, as $t \rightarrow \infty$ we can resort to the ground state dominance principle, which means we can truncate the summation to one leading term:

$$
\begin{aligned}
G\left(\begin{array}{c|c}
r^{\prime}, 0 & r, \theta \\
0 & t
\end{array}\right) & \simeq \frac{1}{2 \pi} \int_{0}^{\infty} e^{-a^{2} \xi_{\mu}^{2} t / 4 B^{2}} \cos (\mu \theta) \times \\
& \times J_{\mu}\left(\xi_{\mu} r / B\right) J_{\mu}\left(\xi_{\mu} r^{\prime} / B\right) d \mu,
\end{aligned}
$$

where $\xi_{\mu}$ is the smallest root of the Bessel function $J_{\mu}(\xi)$.

\section{WINDING ANGLE DISTRIBUTION: SPITZER LAW AND RELATED RESULTS}

\section{A. Winding around a point $(b=0)$}

The authors of the works [1, 2, 4, 5, 6, 7, 8, 9, 10], examined the problem of winding angle distribution in the following formulation. Suppose the walker starts some distance $r$ from the origin, and suppose we are interested in the winding angle distribution irrespective of $r^{\prime}$, the distance from the origin to the trajectory end. Formally, such probability distribution is obtained via suitable integration of the Green function over $r^{\prime}$ :

$$
W(\theta) \propto \int_{0}^{\infty} G\left(\begin{array}{c|c}
r, 0 & r^{\prime}, \theta \\
0 & t
\end{array}\right) r^{\prime} d r^{\prime} .
$$

In the Appendix D we show how to use the Weber integral to follow this path.

Unfortunately, in some other cases considered below, such as winding around a non-zero size disc $(b \neq 0)$, we don't have the advantage of the Weber integral simplification from Eq. (4) to Eq. (5), which makes the explicit integration of the Green function over $r^{\prime}$ difficult. Besides, for polymer applications it is natural to keep track of the end position as long as possible. This is why it is useful to see how we can re-derive the Spitzer law (1) directly from Eq. (4), not resorting to Eq. (5). This is what we shall do now.

We note that integration over $\kappa$ in Eq. (44) is effectively truncated at $\kappa^{2} \leq 4 / t a^{2}$. When $t$ is large enough, this leads to both $\kappa r$ and $\kappa r^{\prime}$ being small. Then the Bessel function can be replaced by the first term of its expansion, $J_{\mu}(\xi) \simeq \frac{1}{\Gamma(1+\mu)}\left(\frac{\xi}{2}\right)^{\mu}$. After that, the integration over $\kappa$ is easily performed, yielding

$$
G\left(\begin{array}{c|c}
r^{\prime}, 0 & r, \theta \\
0 & t
\end{array}\right) \simeq \frac{1}{\pi t a^{2}} \int_{0}^{\infty}\left(\frac{r r^{\prime}}{t a^{2}}\right)^{\mu} \frac{\cos (\mu \theta)}{\Gamma(1+\mu)} d \mu .
$$

Assuming $r r^{\prime} / t a^{2} \ll 1$ (see the discussion a few lines below), we see that the integral over $\mu$ is dominated by small $\mu$ in which area we can set $\Gamma(1+\mu) \simeq 1$. In this approximation, the integration over $\mu$ is elementary, and results in

$$
G\left(\begin{array}{c|c}
r^{\prime}, 0 & r, \theta \\
0 & t
\end{array}\right) \simeq \frac{1}{\pi t a^{2}} \frac{\ln \left(t a^{2} / r r^{\prime}\right)}{\left(\ln \left(t a^{2} / r r^{\prime}\right)\right)^{2}+\theta^{2}} .
$$

This is the Cauchy distribution for the winding angle

$$
W(\theta)=\frac{1}{\pi} \frac{1}{1+x^{2}} ; x=\frac{\theta}{\ln \left(t a^{2} / r r^{\prime}\right)} .
$$

This result is similar, but not identical to the Spitzer formula (11). The difference is in the definition of the scaling variable $x$ : formula (14), unlike (1), keeps track of the coordinates $r$ and $r^{\prime}$ of the trajectory ends. As we have already mentioned, this will be useful for polymer applications. Note, however, that we cannot integrate over $r^{\prime}$ as in Eq. (11), because formula (14) was derived under the assumption that $r^{\prime}$ is not too large.

How then can we recover the Spitzer law (11) from Eq. (14)? What we should do is to note that one trajectory end is fixed at the distance independent of $t$, while the other is free, meaning that $r \sim a$ and $r^{\prime} \sim a \sqrt{t}$. Then, we have for the scaling quantity $x$ in formula (14) $x=$ $\theta / \ln \left(t a^{2} / r r^{\prime}\right) \simeq 2 \theta / \ln t$, which is indeed exactly the same as in Eq (11).

Other interesting extremes are as follows:

- If $r \sim a$ and $r \sim a t$, then the "width" of the distribution gets very small. This is the closest approximation Gaussian model can provide for the idea that fully stretched polymer does not have any freedom to wind around the obstacle. Of course, a Gaussian polymer cannot be fully stretched, this is why, say, $\left\langle\theta^{2}\right\rangle$, remains divergent even when the "width" goes to zero.

- A similar situation is realized when $r \sim r^{\prime} \sim a \sqrt{t}$ : winding is suppressed when the obstacle is removed to the periphery of the coil. Note that Eq. (14) should not be used at larger $r$, when $\kappa r$ is not small and the Bessel function cannot be expanded.

- If both $r \sim a$ and $r^{\prime} \sim a$, then the result is only different from Eq. (11) by a factor of 2 in the definition of $x$; in this case, $x=\theta / \ln t$. That means, fixing both ends and not allowing them to wander freely reduces the "width" by half.

\section{B. Winding around a disc $(b>0)$}

For winding around a disc of finite radius $b$, we can use the same method. When $t$ is large enough, integration over $\kappa$ in Eq. (9) is dominated by small $\kappa$. Accordingly, we can resort to the small $\kappa$ expansion of $Z_{\mu}(\kappa r, \kappa b)$ (see Eq. (B19) and the discussion in the Appendix (B): 


$$
Z_{\mu}(\kappa r, \kappa b) \simeq \frac{(r / b)^{\mu}-(r / b)^{-\mu}}{\sqrt{\left[\left(\frac{\kappa b}{2}\right)^{\mu} \Gamma(1-\mu)-\left(\frac{\kappa b}{2}\right)^{-\mu} \Gamma(1+\mu)\right]^{2}+2 \pi \mu \tan \frac{\pi \mu}{2}}} .
$$

Accordingly, the $\kappa$-dependent factor in the Green function (9) can be presented in the form $e^{-g(\kappa)}$, where

$$
g(\kappa)=\frac{\kappa^{2} t a^{2}}{4}+\ln \left(\left[\left(\frac{\kappa b}{2}\right)^{\mu} \Gamma(1-\mu)-\left(\frac{\kappa b}{2}\right)^{-\mu} \Gamma(1+\mu)\right]^{2}+2 \pi \mu \tan \frac{\pi \mu}{2}\right) .
$$

Provided that $\mu<1$ (which is justified a few lines below), it is not difficult to establish that $g(\kappa)$ has a minimum, which dominates integration over $\kappa$ at large $t$. Straightforward differentiation yields for the the corresponding $\kappa$ the condition

$$
\kappa^{2} t a^{2}=4 \mu \frac{\left(\frac{\kappa b}{2}\right)^{-2 \mu} \Gamma^{2}(1+\mu)-\left(\frac{\kappa b}{2}\right)^{2 \mu} \Gamma^{2}(1-\mu)}{\left(\frac{\kappa b}{2}\right)^{-2 \mu} \Gamma^{2}(1+\mu)+\left(\frac{\kappa b}{2}\right)^{2 \mu} \Gamma^{2}(1-\mu)-2 \pi \mu \cot \pi \mu} .
$$

This equation has just one solution which at large $t$ corresponds to small $\kappa$. More accurately, the solution reads

$$
\frac{\kappa a}{2} \simeq\left\{\begin{array}{ll}
\sqrt{\frac{\mu}{t}} & \text { when } \mu \ln \left(t a^{2} / b^{2}\right) \gg 1 \\
\sqrt{\frac{1}{t \ln \left(t a^{2} / b^{2}\right)}} & \text { when } \mu \ln \left(t a^{2} / b^{2}\right) \ll 1
\end{array} .\right.
$$

As it turns out, the integral over $\mu$ is dominated by $\mu \ll$ $1 / \ln \left(t a^{2} / b^{2}\right)$, so only the lower line of the Eq. (18) is relevant. For small $\mu$, the expression for $Z_{\mu}$ Eq. (15) can be further simplified:

$$
Z_{\mu}(\kappa r, \kappa b) \simeq \frac{\sinh (\mu \ln (r / b))}{\sinh (\mu \ln (2 / \kappa b))} .
$$

Then, replacing $e^{-g(\kappa)}$ with its maximal value, we arrive at the following expression for the Green function:

$$
\begin{aligned}
G\left(\begin{array}{c|c}
r^{\prime}, 0 & r, \theta \\
0 & t
\end{array}\right) & =A \int_{0}^{\infty} d \mu \cos (\mu \theta) \times \\
& \times \frac{\sinh \left(\mu \ln \frac{r}{b}\right) \sinh \left(\mu \ln \frac{r^{\prime}}{b}\right)}{\sinh ^{2}\left(\frac{\mu}{2} \ln \frac{t a^{2}}{b^{2}}\right)}
\end{aligned}
$$

plus some logarithmic corrections. In $A$ we accumulated all the uninteresting constant prefactors, which do not depend on $\theta, r, r^{\prime}$, and $b$.

Now, considering this integral over $\mu$, we have to justify all the assumptions and approximations which we made on the way. First and foremost, the assumption that $\mu$ is small is justified by the rapid convergence of the integral (20). Indeed, at large $\mu$ all three sinh's can be replaced by positive exponentials, leaving us with $\exp \left[-\mu\left(\ln \frac{t a^{2}}{b^{2}}-\ln \frac{r}{b}-\ln \frac{r^{\prime}}{b}\right)\right]$. Since $r r^{\prime} \ll t a^{2}$, the latter two logarithms in the round brackets should be neglected. That means, the convergence of the integral (20) is controlled by the sinh in the denominator, which effectively truncates integration at $\mu$ smaller than $1 / \ln \left(t a^{2} / b^{2}\right)$. This is very good news. First of all, since $1 / \ln \left(t a^{2} / b^{2}\right) \ll 1$, this justifies the small $\mu$ simplification performed in formula (19). Second of all, this also justifies the use of the lower line in the expression (18) for the saddle point. Third, since only small $\mu$ contribute, the validity condition for the expansion of Bessel functions in the first step of Eq. (15), which generally reads $(\kappa r)^{2} \ll 1+\mu$, can be simplified to $r^{2} \ll a^{2} t$ (and similarly $\left.r^{\prime 2} \ll a^{2} t\right)$.

Thus, all approximations leading to the expression (20) are self-consistent. The only task left is to evaluate the integral (20). This task gets easier if we use the notations

$$
\alpha=\frac{2 \pi \ln \frac{r}{b}}{\ln \frac{t a^{2}}{b^{2}}}, \alpha^{\prime}=\frac{2 \pi \ln \frac{r^{\prime}}{b}}{\ln \frac{t a^{2}}{b^{2}}}, x=\frac{2 \theta}{\ln \frac{t a^{2}}{b^{2}}} .
$$

Then, formula (20) is transformed into the following expression for the probability distribution of the winding angle $\theta$, or, better, of the scaling variable $x$, at fixed $r$ and $r^{\prime}$ :

$$
W(\theta)=\frac{\pi}{2 \alpha \alpha^{\prime}} \int_{-\infty}^{+\infty} \frac{\sinh (\xi \alpha / \pi) \sinh \left(\xi \alpha^{\prime} / \pi\right)}{\sinh ^{2} \xi} e^{\imath x \xi} d \xi
$$

where we have re-introduced the normalization factor, such that $\int_{-\infty}^{+\infty} W(\theta) d x=1$. This integral can be reduced to the infinite sum of residues corresponding to the poles along the imaginary axes on the complex $\xi$-plane. In turn, the resulting sum (which is the combination of several geometric series) is easy for Mathematica 33., but can be also computed by hand. One way or the other, here is the result: 
$W(\theta)=\frac{\pi}{2 \alpha \alpha^{\prime}} \frac{\pi x \sinh \pi x \sin \alpha \sin \alpha^{\prime}+\alpha \sin \alpha^{\prime}\left[\cos \alpha^{\prime}-\cos \alpha \cosh \pi x\right]+\alpha^{\prime} \sin \alpha\left[\cos \alpha-\cos \alpha^{\prime} \cosh \pi x\right]}{\left[\cosh \pi x-\cos \left(\alpha-\alpha^{\prime}\right)\right] \times\left[\cosh \pi x-\cos \left(\alpha+\alpha^{\prime}\right)\right]}$

The result (23) is unfortunately quite cumbersome, although it is symmetric and in some ways quite nice. Its beauty is revealed by consideration of various limits. As we learned in the case of point-like obstacle, the most interesting limit is when chain end is free, meaning that $r^{\prime} \sim a \sqrt{t}$. Then, provided only that $t a^{2} \gg b^{2}-$ which is necessary, as the walker must have traveled much farther than the obstacle size $b$, we get $\alpha^{\prime} \simeq \pi$. In this case, we get

$$
W(\theta)=\frac{\pi}{2} \frac{\sin \alpha / \alpha}{\cosh \pi x+\cos \alpha} \stackrel{\alpha \rightarrow 0}{\rightarrow} \frac{\pi / 4}{\cosh ^{2}(\pi x / 2)},
$$

where in the latter transformation we also noted that as the trajectory starting point is fixed, $r$ is independent of $t$, or $\alpha \rightarrow 0$ at large $t$. Thus, we recover formula (2). Importantly, the definition of scaling variable $x$ (21) becomes identical to that in (2), again under the same condition $t a^{2} \gg b^{2}$.

As in the case of point-like obstacle, other interesting extremes are as follows:

- Both $r \sim a \sqrt{t}$ and $r^{\prime} \sim a \sqrt{t}$. In fact, this case is on the border of applicability of our approximations, but qualitatively the result holds. Indeed, $W(\theta)$ becomes very narrow, and approaches $\delta(x)$. This means, no turns are possible around the obstacle which is away from the random walk trajectory.

- Another case, and also a border case in terms of applicability of our approximations, is $r \sim a t$ or $r^{\prime} \sim a t$, implying an exponentially improbable straight trajectory. The distribution is again sharply localized at small $x$.

- Both $r$ and $r^{\prime}$ are independent of $t$, meaning that both $\alpha \simeq 0$ and $\alpha^{\prime} \simeq 0$. This case is safely within the limits of applicability. Then,

$$
W(\theta)=\frac{\pi}{2} \frac{\pi x \sinh \pi x+2(1-\cosh \pi x)}{(1-\cosh \pi x)^{2}} .
$$

As in the $b=0$ case, this distribution, as one can easily check, is exactly two times more narrow than that of (2). In this case, unlike $b=0$, this statement can be formalized by looking at the second moment of the distributions (2) and (25), which (in terms of $x$ ) turns out equal $1 / 3$ and $2 / 3$, respectively.

\section{C. $b \rightarrow 0$ limit: applicability conditions of the Spitzer formula}

According to the Eqs. (11) and (2), winding angle distribution has finite variance at $b \neq 0$ and diverging infinite variance at $b=0$. These equations leave it unclear what happens when the obstacle gets smaller and smaller, or when $b$ decreases and approaches 0 . It is instructive and interesting to use formula (23) to see what really happens when $b \rightarrow 0$.

The important part of our analysis here is to realize that so far we have been using several differently defined scaling variables $x$ : see Eqs. (11), (21), (14), (21). So far, it was (hopefully) clear from the context in every place which $x$ we have in mind. Now, when we examine the $b \rightarrow 0$ limit, we shall face several of these different $x$ simultaneously, so we must be certain as to which $x$ is which. For the rest of this section, we adopt the notation in which each $x$ is labeled with the number of the defining equation: $x_{1}, x_{14}, x_{21}$ (note, that $x_{2}$ is exactly the same as $x_{1} x_{1} \equiv x_{21}$. In particular, $x$ in formula (23) is, of course, $x \sqrt{21}$.

Speaking of different definitions of $x$, we should realize that so far we have been presenting probability distributions $W(\theta)$ normalized with respect to integration over the corresponding $x$. For our purposes now, it is more convenient to use the normalization condition with respect to angle $\theta: \int_{-\infty}^{\infty} W(\theta) d \theta=1$. For the formula (23), this means the factor $2 / \ln \left(t a^{2} / b^{2}\right)$ should be incorporated; we do not re-write the formula for brevity.

The main reason why the difference between various $x$ was unimportant so far is that at $t \rightarrow \infty$ all definitions converge to the same: $x_{14} \rightarrow x_{21} \rightarrow x_{1}=2 \theta / \ln t$. However, when $b \rightarrow 0$, there appears a very broad intermediate range of times $t$ such that although $t a^{2} \gg b^{2}$ (the trajectory is long enough to wind around the obstacle), but $t b^{2} \ll a^{2}$ :

$$
a^{2} / b^{2} \gg t \gg b^{2} / a^{2}
$$

This is the range which we must examine. In this range, to the leading approximation, $x 21$ does not depend on time:

$$
x\left[21=\frac{2 \theta}{\ln \frac{t a^{2}}{b^{2}}} \simeq \frac{\theta}{\ln (a / b)} .\right.
$$

Furthermore, there is a broad range of winding angle $\theta$ in which $x[21$ is small.

Now, we should look at the quantities $\alpha$ and $\alpha^{\prime}$. When $b \rightarrow 0$, both of them turn out to be slightly below $\pi$. For instance,

$$
\alpha=\frac{2 \pi \ln \frac{r}{b}}{\ln \frac{t a^{2}}{b^{2}}}=\pi \frac{\ln \frac{a}{b}+\ln \frac{r}{a}}{\ln \frac{a}{b}+\frac{1}{2} \ln t} \equiv \pi-\delta,
$$

where

$$
\delta=\frac{\ln \frac{t a^{2}}{r^{2}}}{2 \ln \frac{a}{b}} \ll 1
$$


Similarly, $\alpha^{\prime}=\pi-\delta^{\prime}$, with similarly defined $\delta^{\prime} \ll 1$.

Thus, we can simplify formula (23) resorting to expansion of both numerator and denominator over the powers of $x 21, \delta$, and $\delta^{\prime}$. In fact, as we see from Eqs. (2728) all these expansions are ones over the in- verse powers of $\ln (a / b)$, and we keep the leading terms only. Incorporating, as explained above, the factor $2 / \ln \left(t a^{2} / b^{2}\right) \simeq 1 / \ln (a / b)$ to establish the normalization $\int_{-\infty}^{\infty} W(\theta) d \theta=1$, we finally get

$$
\begin{aligned}
& W(\theta) \simeq \frac{1}{\ln \frac{a}{b}} \times \frac{\frac{\pi^{2}}{2}\left(\delta-\delta^{\prime}\right)^{2}\left(\delta+\delta^{\prime}\right)+\frac{\pi^{4}}{2}\left(\delta+\delta^{\prime}\right) x_{[21]}^{2}}{\frac{\pi^{2}}{2}\left(\delta-\delta^{\prime}\right)^{2}\left(\delta+\delta^{\prime}\right)^{2}+\frac{\pi^{4}}{2}\left[\left(\delta+\delta^{\prime}\right)^{2}+\left(\delta-\delta^{\prime}\right)^{2}\right] x \frac{21}{21}+\frac{\pi^{6}}{2} x\left[\frac{4}{21}\right.}=
\end{aligned}
$$

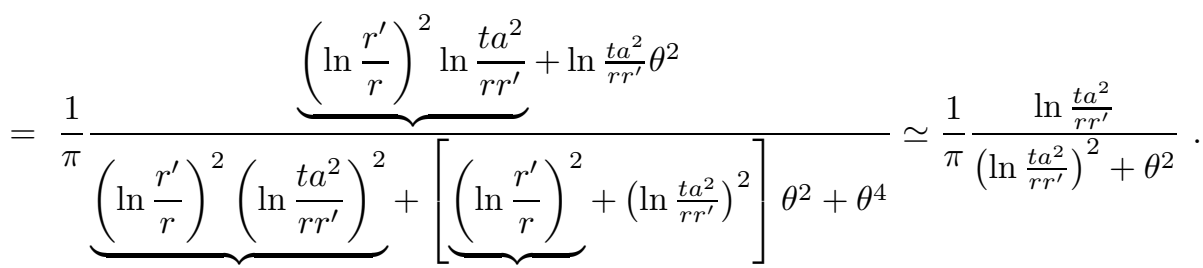

For the two latter steps, we have plugged in the explicit expressions for $\delta, \delta^{\prime}(28)$, and $x$ (27), and then neglected the $\delta-\delta^{\prime} \sim \ln \left(r^{\prime} / r\right)$ terms (which we have underbraced in the intermediate formula). The result is exactly the same as formula (14) (except it is normalized with respect to integration over $\theta$ ).

From our analysis, we can now understand the crossover between Eqs. (2) and (11). Specifically, the Spitzer formula (11) and its generalization (14) apply as long as two conditions are met: $t \ll a^{2} / b^{2}$ and $\theta \ll \ln (a / b)$. At longer times and/or larger angles, the exponential tail of the distribution takes over. For instance, when we say the $\left\langle\theta^{2}\right\rangle$ diverges for winding around a very small obstacle, this really means $\left\langle\theta^{2}\right\rangle \sim(\ln (a / b))^{2}$.

\section{Winding inside a cavity}

We start from Eq. (10). It is not difficult to realize that $\xi_{\mu}$ (the smallest zero of $J_{\mu}(\xi)$ ) increases with $\mu$. Therefore, when $t$ gets large, the integration over $\mu$ is dominated by small $\mu$, as in all previous cases. At small $\mu, \xi_{\mu}$ is a smooth non-singular function, we can linearize it: $\xi_{\mu} \simeq \xi_{0}+\mu \xi_{0}^{\prime}$. Numerically, $\xi_{0} \approx 2.405$ and $\xi_{0}^{\prime} \approx 1.543$. To the same approximation, $J_{\mu}\left(\xi_{\mu} r / B\right) \simeq J_{0}\left(\xi_{0} r / B\right)$. Therefore, evaluation of the integral in Eq. (10) becomes trivial, and the result reads

$$
G\left(\begin{array}{c|c}
r^{\prime}, 0 & r, \theta \\
0 & t
\end{array}\right) \simeq \frac{1}{2} J_{0}\left(\frac{\xi_{0} r}{B}\right) J_{0}\left(\frac{\xi_{0} r^{\prime}}{B}\right) W(\theta),
$$

where the probability distribution of the winding angle is given by

$$
W(\theta)=\frac{1}{\pi} \frac{t a^{2} \xi_{0} \xi_{0}^{\prime} / 2 B^{2}}{\left(t a^{2} \xi_{0} \xi_{0}^{\prime} / 2 B^{2}\right)^{2}+\theta^{2}} .
$$

The decoupling of the ends $r$ and $r^{\prime}$ in formula (31) is not surprising, this is the property of random walk locked in a restricted volume, and it is due to the fact that correlations are broken every time that the trajectory is reflected from the cavity border. As regards probability distribution of winding angle, it is once again the Cauchy distribution, however, the scaling variable involves $\theta / \sqrt{t}$ instead of $\theta / \ln t$ for the random walk in an unrestricted space. This is also because correlations are broken every time that the trajectory hits the border. One can say that pieces of random walk with length about $(B / a)^{2}$ act independently of each other.

This gives rise to the following simple scaling argument providing an insight into the result (31). The winding angle distribution for every "blob" of the length $\sim(B / a)^{2}$ is given by the Spitzer formula (1), with the replacement $t \rightarrow(B / a)^{2}$. Now, we have $t /(B / a)^{2}$ of such blobs. Since blobs are independent, the probability distribution of the sum of all winding angles of all blobs is given as a convolution. In other words, Fourier transform of the Spitzer distribution for one blob, which is $e^{-|\mu| \ln (B / a)^{2}}$, must be taken to the power $t /(B / a)^{2}$. Apart from logarithmic corrections, this returns the result (31).

Thus, the reason why $\left\langle\theta^{2}\right\rangle$ diverges for the polymer inside the cavity is because every blob can make many turns around the point-like obstacle on a small scale, before ever hitting the border of the cavity.

\section{RING POLYMER: EDWARDS-PRAGER-FRISCH MODEL}

We now want to make one step closer to the attempt of gaining insight into the properties of closed ring polymers. One way in this direction would be to say that a ring polymer is the random walk trajectory whose end points happen to coincide, namely $r=r^{\prime}$ and $\theta=2 \pi n$, where integer $n$ (positive, negative, or zero) is the linking number (number of turns). Our results (142325132) are 
suitable for this, and we shall do it. It turns out also useful, however, to derive some additional results independently. In particular, some of the results below are exact (not asymptotically exact, but just exact).

\section{A. Point-like obstacle: a ring with one monomer anchored}

Thus, we return to Eqs. (6 7), and use them this time to write down the statistical weight of the ring polymer conformations with linking number $n$ and with one monomer fixed at the distance $r$ from $\mathcal{O}$ :

$$
\begin{aligned}
G_{n}(r, t) & \equiv G\left(\begin{array}{c|c}
r, 0 & r, 2 \pi n \\
0 & t
\end{array}\right)=\frac{1}{\pi a^{2} t} W_{n}(\xi) ; \\
W_{n}(\xi) & =2 e^{-\xi} \int_{0}^{\infty} \cos (2 \pi n \mu) I_{\mu}(\xi) d \mu ; \quad \xi=\frac{2 r^{2}}{a^{2} t} .
\end{aligned}
$$

In this formula, $G_{n}(r, t)$ is the statistical weight of the ring with $n$ turns, while the prefactor $1 / \pi a^{2} t$ is the statistical weight of a ring with no topological constraints [41. Therefore, $W_{n}(\xi)$ is the probability that polymer ring fixed at one point $r$ makes $n$ turns around the obstacle. In the Appendix E we check explicitly that $W_{n}$ satisfies the normalization condition as a probability.

Similarly to what we did before, we can address the case $t a^{2} \gg r^{2}$, or $\xi \ll 1$. In this case, we truncate the small $\xi$ expansion of $I_{\mu}(\xi) \simeq(\xi / 2)^{\mu} / \Gamma(1+\mu)$, replace $\Gamma(1+\mu) \simeq 1$ (compare Eq. (D1) $)$, and then obtain

$$
W_{n}(\xi) \simeq 2(1-\xi) \frac{\ln (2 / \xi)}{(\ln (2 / \xi))^{2}+4 \pi^{2} n^{2}}, \quad \xi \rightarrow 0 .
$$

Of course, this is nothing else but the "discrete" version of the Spitzer distribution. However, merely taking $\theta=$ $2 \pi n$ in Eq. (14) is not enough, as the normalization factor in (14) corresponds to $\int_{-\infty}^{+\infty} W(\theta) d \theta=1$, while in Eq. (34) it corresponds to $\sum_{n=-\infty}^{+\infty} W_{n}(\xi)=1$.

For a polymer, it makes perfect sense to examine also the opposite extreme, $\xi \gg 1$. The corresponding asymptotics are easy to derive from the somewhat simplified expression for $W_{n}(\xi)$.

We can afford further simplification of the expression Eq. (34) for $W_{n}(\xi)$ resorting to the following integral representation of the modified Bessel function [34]:

$$
\begin{aligned}
I_{\mu}(\xi) & =\frac{1}{2 \pi} \int_{-\pi}^{\pi} e^{\xi \cos \beta} \cos (\beta \mu) d \beta- \\
& -\frac{\sin (\mu \pi)}{\pi} \int_{0}^{\infty} e^{-\xi \cosh u-\mu u} d u
\end{aligned}
$$

which is generally valid at $|\operatorname{Arg} \xi| \leq \pi / 2$ and $\Re \mu>0$. Both of these conditions are met in our case. Substituting this into the Eq. (34), one can easily perform the integration over $\mu$ yielding

$$
W_{n}(\xi)=\Delta_{n 0}+\int_{0}^{\infty} d u e^{-\xi(1+\cosh u)} \times
$$

$$
\times\left[\frac{2 n-1}{u^{2}+\pi^{2}(2 n-1)^{2}}-\frac{2 n+1}{u^{2}+\pi^{2}(2 n+1)^{2}}\right],
$$

where $\Delta_{n 0}$ is the Kronecker symbol ( 1 for $n=0$ and 0 otherwise). The latter result for $n=0$ is worth re-writing separately:

$$
W_{0}(\xi)=1-2 \int_{0}^{\infty} \frac{e^{-\xi(1+\cosh u)}}{u^{2}+\pi^{2}} d u .
$$

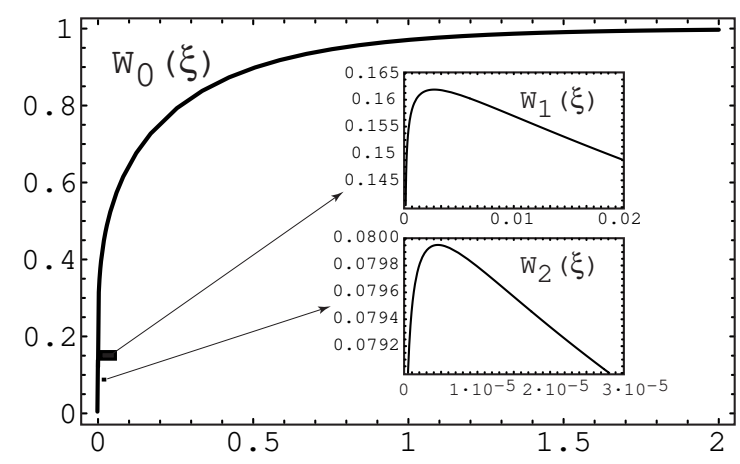

FIG. 2: $W_{n}(\xi)$ is the probability to form a link of order $n$ with the point-like obstacle provided that one of the chain points is fixed at the distance $r$ away from the obstacle, where $\xi=2 r^{2} / a^{2} t, t$ being the chain length. The plots present the result of numerical integration based on formula (36). The plot of $W_{0}(\xi)$ is presented in the main figure. $W_{n}(\xi)$ with $n>0$ would not be seen well in this scale. For both $W_{1}(\xi)$ and $W_{2}(\xi)$, we show the inset, each presenting the vicinity of the maximum; the corresponding places on the main figure are shown by tiny dark rectangles. However small may seem every particular $W_{n}(\xi)$, it should be born in mind that together they sum up to $1-W_{0}(\xi)$.

Equations (3637) are convenient enough to address the $\xi \gg 1$ extreme. Indeed, when $\xi$ is large, the integral converges at small $u$, which allows us to neglect $u$ everywhere except in the exponential factor, where we can also truncate the $\cosh u \simeq 1+u^{2} / 2$. This yields:

$$
W_{n}(\xi) \simeq\left\{\begin{array}{ll}
\frac{\sqrt{2 / \pi^{3}}}{4 n^{2}-1} \frac{e^{-2 \xi}}{\sqrt{\xi}}, & n \neq 0 \\
1-\sqrt{2 / \pi^{3}} \frac{e^{-2 \xi}}{\sqrt{\xi}}, & n=0
\end{array} \quad \xi \rightarrow \infty\right.
$$

Thus, comparing (34) and (38) all $W_{n}(\xi)$ start at 0 at $\xi=$ 0 and grow very rapidly at small $\xi$. At $n=0, W_{0}(\xi)$ keeps increasing monotonically with $\xi$, and $W_{0}(\xi)$ approaches the saturation level of 1 , while all $W_{n}(\xi)$ with $n \neq 0$ decrease and rapidly die away at large $\xi$. Obviously, each of them goes through a maximum. It is not difficult to establish that the maximum of $W_{n}(\xi)$ corresponds to $\xi \sim 1 / \cosh \left(\pi \sqrt{4 n^{2}-1}\right)$ which at large $n$ corresponds to $\xi \sim e^{-2 \pi n}$. This is consistent with the fact that small $\xi$ asymptotics Eq. (34) is valid at $\xi \ll e^{-2 \pi n}$.

Eq. (36) allows also straightforward numerical integration which results in the plots shown in the Fig. 2 


\section{B. Ring polymer entangled with a point}

For the ring polymer, it is not very natural to consider one monomer being fixed at $r$; all monomers of a ring are equivalent. Accordingly, it is natural to define the quantity

$$
\sigma_{n}(t)=\int_{0}^{\infty} W_{n}(\xi) 2 \pi r d r=\frac{\pi a^{2} t}{2} \int_{0}^{\infty} W_{n}(\xi) d \xi .
$$

What is $\sigma_{n}(t)$ ? This quantity has the units of surface area and can be interpreted in the following way. Suppose a ring polymer moves freely on the plane within some large area $A$ (much larger than the polymer size, so the polymer is not restricted in terms of its conformation). Consider one particular conformation of our polymer and then choose a random point $\mathcal{O}$ within $A$. Then $\sigma_{n} / A$ is the probability that polymer makes an $n$-fold link around $\mathcal{O}$. We expect physically that $\sigma_{0}$ should be large, almost as large as $A$. This is also seen directly from the Eq. (37): when integrated over the whole area $A$, the first term (unity) yields just $A$. This is because when $\mathcal{O}$ is outside the coil, there may not be any topological links. If and only if the random point $\mathcal{O}$ is located within the polymer coil can there be any topological link. Therefore, $\sigma_{0}$ should be less than $A$ by a quantity of the order of the coil gyration radius squared, which is of the order $t a^{2}$. On the other hand, $\sigma_{n}$ with $n \neq 0$ should be themselves of order $t a^{2}$, or even smaller.

Another way of understanding of $\sigma_{n}(t)$ is this. Consider one particular conformation of a ring and consider the set of points $\Sigma_{n}$ such that the polymer makes linking with $n$ turns around every point of $\Sigma_{n}$. Then, $\sigma_{n}(t)$ is the surface area, or the measure, associated with the set $\Sigma_{n}$.

Trying to compute $\sigma_{n}(t)$, we can resort to either of the expressions (34) or (36). Let us first explore the first possibility:

$$
\sigma_{n}(t)=\pi t a^{2} \int_{0}^{\infty} e^{-\xi} \int_{0}^{\infty} \cos (2 \pi n \mu) I_{\mu}(\xi) d \mu d \xi
$$

Here, we face a difficulty, because the integral

$$
\int_{0}^{\infty} e^{-\xi} I_{\mu}(\xi) d \xi
$$

diverges at large $\xi$. What is the physical meaning of this? Of course, this is because $\sigma_{0}(t)$ is close to $A$, or, in other words, it is divergent unless we take into account overall volume restriction. We conclude, therefore, that the integral (41) diverges for a good reason: this is because unlinked polymer is free to move away from the obstacle, making $\sigma_{0}$ as large as (almost) $A$.

This hints on the way to circumvent the problem. Let us assume that the polymer is attached to the point $\mathcal{O}$ by a very weak spring. Since such polymer does not move away even when there are no topological links, we expect that even $\sigma_{0}$ should remain finite, independent of $A$. Indeed, instead of (41) we have now

$$
\int_{0}^{\infty} e^{-\alpha \xi} I_{\mu}(\xi) d \xi=\frac{1}{\sqrt{\alpha^{2}-1}\left(\alpha+\sqrt{\alpha^{2}-1}\right)^{\mu}}
$$

which converges at any $\alpha>1$; here $\alpha-1$ is the effective spring constant. Of course, we will take $\alpha \rightarrow 1$ at the end. Performing the remaining integration over $\mu$, we arrive at

$$
\sigma_{n}(t, \alpha)=\frac{\pi t a^{2} \ln \left(\alpha+\sqrt{\alpha^{2}-1}\right)}{\sqrt{\alpha^{2}-1}\left[(2 \pi n)^{2}+\left(\ln \left(\alpha+\sqrt{\alpha^{2}-1}\right)\right)^{2}\right]}
$$

As expected, the $\alpha \rightarrow 1$ limit can now be performed with no difficulties at every $n \neq 0$, yielding finally

$$
\sigma_{n}(t)=\frac{t a^{2}}{4 \pi} \frac{1}{n^{2}}, \quad n \neq 0
$$

Accordingly,

$$
\sigma_{0}(t)=A-\sum_{n \neq 0} \sigma_{n}(t)=A-\frac{\pi}{12} t a^{2} .
$$

That result exactly can be also obtained plugging Eq. (36) into the Eq. (39), although, somewhat surprisingly, calculations are more involved along this route.

We would like to remind to our reader once again, that $\sigma_{n}(t) / A$ is the probability to have linking number $n$ for the polymer of the length $t$. As the probability distribution, $\sigma_{n}$ has the peculiarity that all its moments obviously diverge, even just the average linking number is infinite. It is not difficult to trace this back to the fact that infinitely flexible polymer, as represented by the Brownian random walk trajectory, can make infinitely many turns around a point-like obstacle. We shall address this further later.

It is worth emphasizing that the results (4445) are exact, their validity does not require even that $t$ is large - they are exact at any $t$.

\section{How far is the ring from the point-like obstacle?}

One more interesting quantity to look at is $\left\langle r^{2}\right\rangle$ : the mean squared distance of one particular point on the ring to the obstacle, $\mathcal{O}$. To determine the probability distribution for $r$, we note that $\sigma_{n}(t)$ plays the role of a partition function. The probability density for $r$ reads

$$
\frac{G\left(\begin{array}{c|c}
0, r & 2 \pi n, r \\
0 & t
\end{array}\right)}{\int_{0}^{\infty} G\left(\begin{array}{c|c}
0, r & 2 \pi n, r \\
0 & t
\end{array}\right) 2 \pi r d r}=\frac{W_{n}(\xi)}{\sigma_{n}(t)}
$$

When computing $\left\langle r^{2}\right\rangle$ from this, formula (43) comes in handy, as $\left\langle r^{2}\right\rangle$ is basically the derivative of $\sigma_{n}(t, \alpha)$ with 
respect to $\alpha$ at $\alpha=1$ :

$$
\begin{aligned}
\left\langle r^{2}\right\rangle & =\frac{\pi}{\sigma_{n}(t)}\left(\frac{a^{2} t}{2}\right)^{2} \int_{0}^{\infty} W_{n}(\xi) \xi d \xi= \\
& =-\left.\frac{a^{2} t}{2 \sigma_{n}(t)} \frac{\partial \sigma_{n}(t, \alpha)}{\partial \alpha}\right|_{\alpha=1} .
\end{aligned}
$$

Straightforward calculation yields

$$
\left\langle r^{2}\right\rangle=\frac{a^{2} t}{6}\left[1+\frac{3}{2 \pi n^{2}}\right] .
$$

The result is interesting. Surprisingly, it goes to a finite constant proportional to the unperturbed coil size $t a^{2}$ in the limit of very strong linking, $n \rightarrow \infty$. This should be understood by noting that even very large number of turns will be produced by a short piece of a trajectory, leaving a long part, of the order $t$, unentangled, with the size of order $a^{2} t$.

This is reminiscent of the recent findings by Kardar and his co-workers [35] in which they claim that in many cases real knots in three dimensional polymeric loops are entropically dominated by conformations with the knot tightened in a short piece of polymer, and with the rest of the polymer fluctuating freely with no knots.

\section{Force}

When winding model was first introduced in the polymer physics 14, 15, 16], it was done mostly in connection with problems related to the rubber elasticity. Accordingly, elastic force, or force-extension curve, was the primary subject of interest. In case of DNA, such a curve can be also measured using some sort of a single molecule technique [36]. Although both in rubbers and in DNAs real forces have both entropic and enthalpic contributions, in the winding model the force has purely entropic nature and, therefore, it is proportional to $k_{B} T$ in standard notations, where $k_{B}$ is Boltzmann constant and $T$ is absolute temperature. In our notations, the force $f_{n}$ which should be applied to the polymer to keep one of its links a certain distance $r$ from the obstacle $\mathcal{O}$ at the fixed topological invariant $n$ is given by

$$
\frac{f_{n}}{k_{B} T}=-\frac{\partial \ln W_{n}}{\partial r}=-\frac{4 r}{t a^{2} W_{n}} \frac{\partial W_{n}}{\partial \xi} .
$$

We have not found any simple closed expression for the force, in this sense we make really no progress on this point compared to the papers [14, 15, 16]. Nevertheless, qualitatively, one glance at the Figure 2 is sufficient to realize that the force $f_{0}$ of an unentangled ring is always positive. This is obviously because this ring is topologically repelling the obstacle. On the other hand, when $n \neq 0$, the force is positive, corresponds to repulsion, only when $r$ (or $\xi$ ) is small enough. At larger $r$ (or $\xi$ ), the force flips sign and becomes negative, which obviously corresponds to the elastic stress caused in the polymer ring by an attempt to pull it away from the obstacle with which the ring is entangled.

\section{E. Ring polymer entangled with a finite size disc}

For the obstacle of finite radius $b$, we were unable to obtain exact answers similar to Eqs. (44) or (48). All we can do for this case is to resort to the asymptotic calculations. One of the advantages of the finite size obstacle model is that it allows to examine both asymptotics, we call them loose entanglement and tight entanglement, respectively. The former regime is realized when the size of the obstacle $b$ is smaller than typical polymer coil dimension $a t^{1 / 2}$, and, moreover, when minimal length necessary to make $n$ turns, $2 \pi b n$, is still small compared to $a t^{1 / 2}: n b \ll a t^{1 / 2}$. In this case, calculations are similar to those of Section IVB In the opposite extreme, when $n b \gg a t^{1 / 2}$, polymer has to be significantly stretched out to make all $n$ turns. This corresponds to the far tail of winding angle distribution, which is usually not examined and which we did not consider in Section IVB

\section{Loose entanglement}

All we can do for this case is to resort to the asymptotic calculations similar to those of Section IVB In fact, the calculations are almost identical, and at the end they return essentially the result (25), with the only difference in the normalization factor. Specifically, the probability to have linking number $n$ is proportional to

$$
W_{n} \propto \frac{\pi x_{n} \sinh \pi x_{n}+2\left(1-\cosh \pi x_{n}\right)}{\left(1-\cosh \pi x_{n}\right)^{2}} .
$$

where the omitted normalization factor must be defined such that $\sum_{n=-\infty}^{\infty} W_{n}=1$, and where

$$
x_{n}=\frac{4 \pi n}{\ln \frac{t a^{2}}{b^{2}}} .
$$

Similarly, although we cannot find the exact expression for the value of $\sigma_{n}(t)$, but the estimate reads $\sigma_{n}(t) \sim$ $\left[\pi b^{2}+r^{2} W_{n}\right]_{r \sim a \sqrt{t}}$.

Thus, quantities such as $W_{n}$ and $\sigma_{n}(t)$ decay exponentially at very large $n$, and the characteristic $n$ where exponential decay starts is about $\ln \left(t a^{2} / b^{2}\right)$. This latter quantity estimates also the characteristic linking number in another sense, defined as the root mean squared, $\sqrt{\left\langle n^{2}\right\rangle}$. This is an interesting and somewhat unexpected result. Indeed, one could have naively expected that the characteristic value of $n$ should be proportional to $t a^{2} / b^{2}$. Indeed, we expect that one turn around the obstacle should be similar to walking a distance about $2 \pi b \sim b$, which requires a time $\tau \sim b^{2} / a^{2}$, implying the number of turns to be about $t / \tau$. Instead, we are getting something like $\ln (t / \tau)$. This happens because a large portion of the chain length deviates much further away from the obstacle than $b$, and it makes turns around a much larger circumference. This once again suggests that knot tightening [35] occurs even in this case of a disc-like obstacle with excluded volume. 
Similar to our discussion in section IVC we can understand what happens when $b \rightarrow 0$. In this case, there appears a wide interval of polymer chain lengths $b^{2} / a^{2} \ll t \ll a^{2} / b^{2}$ in which, say, $\sigma_{n}(t)$ decays only as $1 / n^{2}$ in the wide interval of $n$, up to a large value of $n$ of about $\ln (a / b)$.

\section{Tight entanglement}

The tight entanglement regime is realized when $n^{2} b^{2} \gg$ $t a^{2}$. In this case, polymer barely has enough length to make $n$ turns around the obstacle. Obviously, the dominant polymer conformations are those tightly wound around the obstacle. This regime is similar to ray optics 38. Indeed, if one searches for the solution of diffusion equation (3) in the form $G=\exp [s(\vec{r}, t)]$ and assumes that $s$ is (in a proper sense) a slowly changing function, then the so-called eikonal equation for $s$ results:

$$
-\frac{\partial s}{\partial t}=\frac{a^{2}}{6}(\vec{\nabla} s)^{2}
$$

For the system at hand, namely a polymer with winding angle $\theta$ around the obstacle of radius $b$, this equation allows for the exact solution:

$$
s=\frac{3}{2 a^{2}} \frac{L^{2}}{t}
$$

where $L$ is the shortest distance between fixed ends consistent with the topological constraint (that is, with the given winding angle):

$$
L=b\left(\theta-\vartheta-\vartheta^{\prime}\right)+\sqrt{r^{2}-b^{2}}+\sqrt{{r^{\prime}}^{2}-b^{2}} .
$$

Here, $\vartheta$ and $\vartheta^{\prime}$ are determined by the conditions $\cos \vartheta=$ $b / r$ and $\cos \vartheta^{\prime}=b / r^{\prime}$. Both these conditions and the solution itself are quite easy to establish based on the geometry presented in the figure 3 It is also easy to check by direct differentiation that formulas (53154) present an exact solution of the eikonal equation (52).

\section{F. Ring polymer inside a cavity}

Our discussion in the previous section is additionally illuminated by the problem of a ring polymer entangled with an obstacle while confined in a cavity of the radius $B$. In this case, the result (32) directly applies, apart from the replacement $\theta \rightarrow 2 \pi n$, and the proper normalization factor. We see that in this case the characteristic value of $n$ is proportional to $t a^{2} / B^{2}$. This number can be understood by saying that polymer is confined to a tube of the width $D=B$ and length $L=n B$, where the typical $n$ must be determined such that confinement entropy is similarly contributed by chain squeezing across the tube and stretching along the tube (compare similar arguments in [39]). The confinement entropy is well

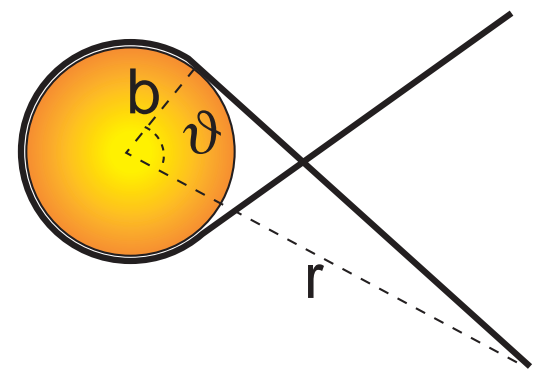

FIG. 3: Tight entanglement, or "ray optics" limit. In this figure, for the ease of drawing, we assume that the polymer makes just a little more than one turn around the obstacle, while its ends are fixed at the given points. Distance to one end $r$ and the corresponding angle $\vartheta$ are shown in the figure; similar distance $r^{\prime}$ and angle $\vartheta^{\prime}$ are not shown to simplify the figure.

known [40], it is proportional to $t a^{2} / D^{2}+L^{2} / t a^{2}$, where the two terms correspond to the two factors just mentioned - chain squeezing across the tube and stretching along the tube, respectively. Equating the two terms, we arrive at $n \sim t a^{2} / B^{2}$, as expected.

\section{CONCLUDING REMARKS}

We have focused on a special application, a toy Edwards-Prager-Frisch model [14, 15], and its generalization. It can be viewed as a model of an unsymmetrical infinite catenane. This catenane is formed from a random walk of $t$ steps and "entwined" with infinite, rigid, closed structure composed of two straight legs, which are separated at least by a distance larger than $t a$, meeting at infinity. As such it allows one to extrapolate a limiting probability of catenation by a closed random walk, which is consistent with earlier estimates.

This model is widely recognized as the simplest playground for "statistical mechanics with topological constraints" 14]. Unfortunately, no simple notable result had previously come out of this model studies - except the very fact that it is "exactly solvable." We were lucky to find a couple of such simple results. First, looking at the entanglement as an element of "annealed" disorder, we found that the area associated with all points around which closed random walk makes exactly $n$ turns is equal to $\left\langle R^{2}\right\rangle / 4 \pi n^{2}$, where $\left\langle R^{2}\right\rangle$ is the mean-square end-to-end distance of the linear walk of the same length. Second, looking at the entanglement as an element of "quenched" disorder, we found that the mean-squared distance between an obstacle an an arbitrary monomer of an $n$ times entangled ring is equal to $(1 / 6)\left\langle R^{2}\right\rangle\left[1+3 / 2 \pi n^{2}\right]$. Both results are exact. We have also found that the entanglement of a very long polymer is very uneven, in the sense that it tends to segregate into one very long loop, almost as long as the entire polymer, and a number of much shorter loops.

The generalization of Edwards-Prager-Frisch model, in 
which an obstacle is not a point, but a disc of finite radius $b$, as far as we could tell, does not allow for an exact solution in any useful closed form. However, we were able to show that for annealed loop the mean-squared linking number turns out to be finite, of order $\left\langle n^{2}\right\rangle \sim \ln (a / b)$. As $b$ increases, the entanglement becomes increasingly tight, resulting in all loops being of comparable length.

Our deliberations clearly reflect three aspects: The first is the fruitful nature of Spitzer's 1] original insight for both further theory and its applications. The second is the value of self-consistent approximations in the field when direct exact calculations are precluded by mathematical difficulty. And, last but not least, the third is the usefulness of complete analysis of those simplified models which do allow for mathematically exact solutions.

\section{Acknowledgments}

The AG work was supported in part by the MRSEC Program of the NSF under Award Number DMR0212302. Part of this work was performed by AG while visiting Kavli Institute for Theoretical Physics, University California - Santa Barbara; this part was supported by the National Science Foundation under Grant No. PHY99-07949. Preprint of this work is published as NSFKITP-03-044. HF acknowledges the support under NSF grant DMR- 9628224.

\section{APPENDIX A: WEBER INTEGRAL}

First let us prove an auxiliary relation:

$$
\int e^{-\alpha^{2}\left(\vec{r}-\vec{r}^{\prime}\right)^{2}} \psi_{\kappa}\left(\vec{r}^{\prime}\right) d^{2} \vec{r}^{\prime}=\frac{\pi}{\alpha^{2}} e^{-\kappa / 4 \alpha^{2}} \psi_{\kappa}(\vec{r}),
$$

where $\psi_{\kappa}(\vec{r})$ is an eigenfunction of the Laplacian operator corresponding to the eigenvalue $-\kappa^{2}: \Delta \psi=-\kappa^{2} \psi(\vec{r})$. To see this, we note that $\left(\alpha^{2} / \pi\right) e^{-\alpha^{2}\left(\vec{r}-\vec{r}^{\prime}\right)^{2}}$ is the Green function of the diffusion equation in which $\alpha^{2}$ plays the role of $1 / D t$, with $D$ and $t$ being diffusion coefficient and time, respectively. Therefore, this exponent can be written in terms of a bilinear expansion

$$
\frac{\alpha^{2}}{\pi} e^{-\alpha^{2}\left(\vec{r}-\vec{r}^{\prime}\right)^{2}}=\sum_{k} \psi_{k}(\vec{r}) \psi_{k}\left(\vec{r}^{\prime}\right) e^{-k^{2} / \alpha^{2}},
$$

where the summation runs over the entire spectrum of the Laplacian operator. By making a dot-product of both sides with $\psi_{\kappa}$, we arrive at the result A1.

Let us now use the formula (A1) choosing $\psi_{\kappa}=$ $J_{n}(\kappa r) e^{\imath n \theta}$. At any $n$, this is indeed one of the eigenfunctions of the Laplacian operator, corresponding to the eigenvalue $-\kappa^{2}$. Now, we make use of the following relation

$$
\int_{0}^{2 \pi} e^{\alpha^{2} \cos \phi+\imath \mu \phi} d \phi=2 \pi I_{\mu}\left(\alpha^{2}\right)
$$

which is most frequently encountered as an integral representation of the modified Bessel function $I_{\mu}$. This relation leads to

$$
\begin{aligned}
\int_{0}^{\infty} J_{\mu}(\kappa y) I_{\mu}\left(2 \alpha^{2} x y\right) & \times e^{-\alpha^{2} y^{2}} y d y= \\
& =e^{\alpha^{2} x^{2}-\frac{\kappa^{2}}{4 \alpha^{2}}} \frac{J_{\mu}(\kappa x)}{2 \alpha^{2}} .
\end{aligned}
$$

Changing variables, we finally obtain the two formulations of the Weber integral:

$$
\begin{aligned}
\int_{0}^{\infty} J_{\mu}(\kappa y) I_{\mu}\left(\kappa^{\prime} y\right) & \times e^{-\alpha^{2} y^{2} y d y=} \\
& =\frac{e^{\frac{\kappa^{\prime 2}-\kappa^{2}}{4 \alpha^{2}}}}{2 \alpha^{2}} J_{\mu}\left(\frac{\kappa \kappa^{\prime}}{2 \alpha^{2}}\right) \\
\int_{0}^{\infty} J_{\mu}(\kappa y) J_{\mu}\left(\kappa^{\prime} y\right) & \times e^{-\alpha^{2} y^{2}} y d y= \\
& =\frac{e^{-\frac{\kappa^{\prime 2}+\kappa^{2}}{4 \alpha^{2}}}}{2 \alpha^{2}} I_{\mu}\left(\frac{\kappa \kappa^{\prime}}{2 \alpha^{2}}\right)
\end{aligned}
$$

The latter formula is what needs to be used to go from Eq. (4) to Eq. (5).

\section{APPENDIX B: SOME PROPERTIES OF THE FUNCTIONS $Z$}

\section{Orthogonality}

First of all, we want to prove here that the functions $Z_{\mu}(\kappa r, \kappa b)$, as defined by the Eq. (요), are orthogonal and normalized:

$$
\begin{aligned}
\int_{b}^{\infty} Z_{\mu}(\kappa r, \kappa b) Z_{\mu}\left(\kappa^{\prime} r, \kappa^{\prime} b\right) r d r & =\frac{1}{\kappa} \delta\left(\kappa-\kappa^{\prime}\right) \\
\int_{0}^{\infty} Z_{\mu}(\kappa r, \kappa b) Z_{\mu}\left(\kappa r^{\prime}, \kappa b\right) \kappa d \kappa & =\frac{1}{r} \delta\left(r-r^{\prime}\right)
\end{aligned}
$$

Note that when $b \rightarrow 0$, we have $Z_{\mu}(\kappa r, \kappa b) \simeq J_{\mu}(\kappa r)$ (because in this case $Y_{\mu}(\kappa b)$ is negative and large in absolute value), so in this limit both equations (B1) and (B2) come back to the well known relation [37]

$$
\int_{0}^{\infty} J_{\mu}(\kappa r) J_{\mu}\left(\kappa^{\prime} r\right) r d r=\frac{1}{\kappa} \delta\left(\kappa-\kappa^{\prime}\right) .
$$

In what follows, we derive the relations (B1 B2). For simplicity of notations, it is easier to compute the normalization of the functions

$$
U_{\mu}(\kappa r, \kappa b)=-J_{\mu}(\kappa r) Y_{\mu}(\kappa b)+J_{\mu}(\kappa b) Y_{\mu}(\kappa r)
$$

from which the properties of $Z$ will follow automatically. Derivation consists of two parts, one of them is trivial, and the other is only slightly less trivial. 


\section{a. Trivial part}

To begin with, there is a useful general formula

$$
\int U_{\mu}^{2}(\kappa r, \kappa b) r d r=\frac{\kappa^{2} r^{2}-\mu^{2}}{2 \kappa^{2}} U_{\mu}^{2}+\frac{r^{2}}{2 \kappa^{2}}\left(\frac{\partial U_{\mu}}{\partial r}\right)^{2},
$$

which is valid for any solution of Bessel equation, that is, for any linear combination of $J_{\mu}(\kappa r)$ and $Y_{\mu}(\kappa r)$, including the $U_{\mu}$. The derivation of this formula can be found in many places, for instance, 37]. One way is to take $\left(r \partial U_{\mu} / \partial r\right)^{2}$ and differentiate it over $r$. Remembering that $U_{\mu}$ satisfies Bessel equation, it is easy to find

$$
\frac{\partial}{\partial r}\left(r \frac{\partial U_{\mu}}{\partial r}\right)^{2}=2 r^{2}\left[\frac{\mu^{2}}{r^{2}}-\kappa^{2}\right] U_{\mu} \frac{\partial U_{\mu}}{\partial r}
$$

from which Eq. (B5) follows automatically.

We cannot directly apply this formula for the case of an infinite interval, because it yields the divergence (due to the $r^{2} U_{\mu}^{2}$ term: $U_{\mu}$ decays only as $1 \sqrt{r}$ at large $r$ ). Indeed, this is not surprising, since the answer (B1) contains a $\delta$-function. Thus, what we shall do is to consider first the finite width ring $b<r<B$, with boundary condition $\left.U_{\mu}\right|_{r=B}=0$. In the end, we shall send $B \rightarrow \infty$.

Assuming $\left.U_{\mu}(\kappa r, \kappa b)\right|_{r=B}$, we get from (B5)

$$
\int_{b}^{B} U_{\mu}^{2}(\kappa r, \kappa b) r d r=\left[\frac{r^{2}}{2 \kappa^{2}}\left(\frac{\partial U_{\mu}}{\partial r}\right)^{2}\right]_{b}^{B} .
$$

The derivative $\partial U_{\mu} / \partial r$ can be simplified, because it is related to the Wronskian of $J_{\mu}(\kappa r)$ and $Y_{\mu}(\kappa r)$, which is equal to $2 / \pi \kappa r$. Taking into account the boundary condition

$$
Y_{\mu}(\kappa b) J_{\mu}(\kappa B)=Y_{\mu}(\kappa B) J_{\mu}(\kappa b),
$$

we obtain

$$
\begin{aligned}
\int_{b}^{B} U_{\mu}^{2}(\kappa r, \kappa b) r d r & =\frac{2}{\kappa^{2} \pi^{2}}\left[\frac{J_{\mu}^{2}(\kappa b)}{J_{\mu}^{2}(\kappa B)}-1\right]= \\
& =\frac{2}{\kappa^{2} \pi^{2}}\left[\frac{Y_{\mu}^{2}(\kappa b)}{Y_{\mu}^{2}(\kappa B)}-1\right] .
\end{aligned}
$$

If we have two different values, $\kappa \neq \kappa^{\prime}$, both satisfying boundary condition (B8), then

$$
\int_{b}^{B} U_{\mu}(\kappa r, \kappa b) U_{\mu}\left(\kappa^{\prime} r, \kappa^{\prime} b\right) r d r=0
$$

as can be established either by proper integration by parts using the Bessel equation, or by direct reference to the fact that these $U$ 's are the eigenfunctions of a Hermitian operator belonging to different eigenvalues. Thus, we can use the Kroneker symbol to write

$$
\begin{aligned}
& \int_{b}^{B} U_{\mu}(\kappa r, \kappa b) U_{\mu}\left(\kappa^{\prime} r, \kappa^{\prime} b\right) r d r= \\
& =\frac{2}{\kappa^{2} \pi^{2}}\left[\frac{J_{\mu}^{2}(\kappa b)}{J_{\mu}^{2}(\kappa B)}-1\right] \Delta_{\kappa \kappa^{\prime}} .
\end{aligned}
$$

Here, we sacrificed the beauty of symmetry and used the upper line of the Eq. (B9); the same final answer is obtained from the lower line.

\section{b. Slightly less trivial part}

We have to perform now the limit $B \rightarrow \infty$. The difficulty is that when $B$ changes, so does also $\kappa$, since it is subject to boundary condition (B8). To circumvent this problem, the following trick is suggested. Let us choose some particular value of $\kappa$, then boundary condition (B8) is satisfied by some discrete set of $B$ values. Let us send $B \rightarrow \infty$ stepping over these specific values and thus keeping $\kappa$ fixed. Then, when $B$ is already large enough, we can resort to the well known asymptotics

$$
\begin{aligned}
& J_{\mu}(x) \simeq \sqrt{\frac{2}{\pi x}} \cos \left[x-\frac{\pi}{2}\left(\mu+\frac{1}{2}\right)\right], \\
& Y_{\mu}(x) \simeq \sqrt{\frac{2}{\pi x}} \sin \left[x-\frac{\pi}{2}\left(\mu+\frac{1}{2}\right)\right] .
\end{aligned}
$$

Then, formula (B11) yields

$$
\begin{aligned}
& \int_{b}^{B} U_{\mu}(\kappa r, \kappa b) U_{\mu}\left(\kappa^{\prime} r, \kappa^{\prime} b\right) r d r= \\
& \quad=\frac{2}{\kappa^{2} \pi^{2}}\left[\frac{\pi \kappa B}{2} \frac{J_{\mu}^{2}(\kappa b)}{\cos ^{2}\left[\kappa B-\frac{\pi}{2}\left(\mu+\frac{1}{2}\right)\right]}-1\right] \Delta_{\kappa \kappa^{\prime}} .
\end{aligned}
$$

On the other hand, we can also use asymptotics (B12) for $J_{\mu}(\kappa B)$ and for $Y_{\mu}(\kappa B)$ to simplify the boundary condition (B8); by some easy manipulations, we can re-write this boundary condition in the form

$$
1+\left[\frac{Y_{\mu}(\kappa b)}{J_{\mu}(\kappa b)}\right]^{2}=\frac{1}{\cos ^{2}\left[\kappa B-\frac{\pi}{2}\left(\mu+\frac{1}{2}\right)\right]} .
$$

Then, formula (B13) yields to the leading order in $B$ :

$$
\begin{aligned}
& \int_{b}^{B} U_{\mu}(\kappa r, \kappa b) U_{\mu}\left(\kappa^{\prime} r, \kappa^{\prime} b\right) r d r= \\
& \quad=\frac{B}{\kappa \pi}\left[J_{\mu}^{2}(\kappa b)+Y_{\mu}^{2}(\kappa b)\right] \Delta_{\kappa \kappa^{\prime}}
\end{aligned}
$$

Finally, we argue that at large $B$ the Kronecker $\Delta$ should be replaced with Dirac $\delta$ according to

$$
\Delta_{\kappa \kappa^{\prime}} \rightarrow \frac{\pi}{B} \delta\left(\kappa-\kappa^{\prime}\right) .
$$

To make this conclusion, we switch to the view point in which $B$ can be arbitrary, while $-\kappa^{2}$ and $-\kappa^{\prime 2}$ are the eigenvalues which depend on $B$. Then, when $B \rightarrow \infty$, the eigenvalues come closer to one another, with the interval between neighboring $\kappa$ equal to $\pi / B$, as it is clear from the asymptotics of Bessel functions (B12). Therefore, 
any sum involving Kroneker $\Delta$ can be transformed into the integral

$$
\sum_{\kappa^{\prime}} \ldots \Delta_{\kappa \kappa^{\prime}} \rightarrow \int \ldots \Delta_{\kappa \kappa^{\prime}} \frac{d \kappa^{\prime}}{\pi / B}
$$

which means precisely (B16).

Taken together, equations $(\mathrm{B} 15)$ and $(\mathrm{B} 16)$ yield the answer

$$
\int_{b}^{\infty} U_{\mu}(\kappa r, \kappa b) U_{\mu}\left(\kappa^{\prime} r, \kappa^{\prime} b\right) r d r=\frac{J_{\mu}^{2}(\kappa b)+Y_{\mu}^{2}(\kappa b)}{\kappa} \delta\left(\kappa-\kappa^{\prime}\right)
$$

which is essentially formula (B1). Formula (B2) follows automatically from $(\mathbb{B 1})$ and the the fact that functions $Z_{\mu}$ form a complete set, which, in turn, follows from the very general spectral consideration.

\section{Asymptotics of $Z$}

Here, we first briefly describe the derivation of the small $\kappa$ asymptotics of $Z_{\mu}(\kappa r, \kappa b)$, Eq. (15). Knowing that 37 .

$$
\begin{aligned}
& J_{\mu}(\xi) \simeq \frac{(\xi / 2)^{\mu}}{\Gamma(1+\mu)} \\
& Y_{\mu}(\xi) \simeq \frac{(\xi / 2)^{\mu}}{\Gamma(1+\mu)} \cot \pi \mu-\frac{(2 / \xi)^{\mu}}{\Gamma(1-\mu)} \frac{1}{\sin \pi \mu}
\end{aligned}
$$

at $\xi^{2} \ll 1+\mu$, and using the identity

$$
\Gamma(1+\mu) \Gamma(1-\mu) \sin \pi \mu=\pi \mu,
$$

and not resorting to any further approximations, we arrive at the Eq. (15).
For completeness, we also mention the large $\kappa$ asymptotics of $Z$, which turn out to be particularly nice:

$$
Z_{\mu}(\kappa r, \kappa b) \simeq \sqrt{\frac{2}{\pi \kappa r}} \sin (\kappa(b-r)) .
$$

\section{APPENDIX C: ALTERNATIVE REPRESENTATION OF THE GREEN'S FUNCTION}

Consider again the diffusion equation, which at $t>t^{\prime}$ reads

$$
\frac{\partial G}{\partial t}=\frac{a^{2}}{4} \Delta G
$$

subject to the initial and the boundary conditions

$$
\left.G\right|_{t=0}=\delta\left(\vec{r}-\vec{r}^{\prime}\right),\left.\quad G\right|_{r=b}=0,\left.\quad G\right|_{r \rightarrow \infty} \rightarrow 0 .
$$

Let us denote

$$
G_{p, \mu}\left(r, r^{\prime}\right)=\int_{0}^{\infty} e^{-p t} \int_{-\infty}^{+\infty} e^{\imath \mu \theta} G\left(t, \vec{r}, \vec{r}^{\prime}\right) d \theta d t .
$$

This satisfies

$$
\begin{aligned}
\frac{1}{r^{2}} \frac{\partial}{\partial r} r^{2} \frac{\partial}{\partial r} G_{p, \mu}\left(r, r^{\prime}\right) & -\left[\frac{4 p}{a^{2}}+\frac{\mu^{2}}{r^{2}}\right] G_{p, \mu}\left(r, r^{\prime}\right)= \\
& =-\frac{4}{r a^{2}} \delta\left(r-r^{\prime}\right)
\end{aligned}
$$

The solution of this equation is the linear combinations of the Bessel functions $K_{\mu}$ and $I_{\mu}$. Making it to satisfy the boundary conditions at $r=0, r \rightarrow \infty$, and at $r=r^{\prime}$ (the latter dictated by the $\delta$-function), one arrives at

$$
G_{p, \mu}\left(r, r^{\prime}\right)=\left\{\begin{array}{l}
\frac{4}{a^{2}} \frac{K_{\mu}\left(\frac{2 r^{\prime} \sqrt{p}}{a}\right)}{K_{\mu}\left(\frac{2 b \sqrt{p}}{a}\right)}\left[I_{\mu}\left(\frac{2 r \sqrt{p}}{a}\right) K_{\mu}\left(\frac{2 b \sqrt{p}}{a}\right)-I_{\mu}\left(\frac{2 b \sqrt{p}}{a}\right) K_{\mu}\left(\frac{2 r \sqrt{p}}{a}\right)\right] \text { when } r<r^{\prime} \\
\frac{4}{a^{2}} \frac{K_{\mu}\left(\frac{2 r \sqrt{p}}{a}\right)}{K_{\mu}\left(\frac{2 b \sqrt{p}}{a}\right)}\left[I_{\mu}\left(\frac{2 r^{\prime} \sqrt{p}}{a}\right) K_{\mu}\left(\frac{2 b \sqrt{p}}{a}\right)-I_{\mu}\left(\frac{2 b \sqrt{p}}{a}\right) K_{\mu}\left(\frac{2 r^{\prime} \sqrt{p}}{a}\right)\right] \text { when } r>r^{\prime}
\end{array} .\right.
$$

As expected, this is the symmetric function of $r$ and $r^{\prime}$. This formula was already obtained in [5] (equation (2.9) of that work).

What we should do now is to invert the respective Laplace and Fourier transforms:

$$
G\left(r, r^{\prime}, \theta, t\right)=\frac{1}{(2 \pi)^{2} \imath} \int_{-\infty}^{\infty} \int_{\mathcal{C}} e^{-\imath \mu \theta} e^{p t} G_{p, \mu}\left(r, r^{\prime}\right) d p d \mu
$$

where $\mathcal{C}$ is the vertical contour in the plane of complex variable $p$ which should be to the right of all singularities of $G_{p, \mu}$. Knowing the explicit expression of $G_{p, \mu}$, Eq. (C5), we see that it has the singularity at $p=0$. This singularity is due to both the branch point of $\sqrt{p}$ and the singular behavior of many Bessel functions at zero. Then, it is convenient to place the branch cut along the negative real axis in complex $p$-plane, and then to deform the contour from $\mathcal{C}$ to $\mathcal{C}_{1}$, as shown in the Figure 
4. Then, because of the branch cut, on the lower side of the contour $\mathcal{C}_{1}$ we have $p=e^{-\imath \pi}|p|$, while on the upper side we have $p=e^{\imath \pi}|p|$. Furthermore, instead of $|p|$, it is convenient to introduce the new variable, $\kappa$, such that $|p|=\kappa^{2} a^{2} / 4$. Then, integrals along the lower and along the upper sides of the contour $\mathcal{C}_{1}$ are each represented by integration from 0 to $\infty$ over $\kappa$. We can combine these two integrals together, and then simple algebra yields

$$
\begin{aligned}
G\left(r, r^{\prime}, \theta, t\right) & =\frac{2 \imath}{(2 \pi)^{2}} \int_{-\infty}^{\infty} \int_{0}^{\infty} e^{-\imath \mu \theta} e^{-\kappa^{2} a^{2} t / 4}\left\{\frac{K_{\mu}\left(\imath \kappa r^{\prime}\right)}{K_{\mu}(\imath \kappa b)}\left[I_{\mu}(\imath \kappa r) K_{\mu}(\imath \kappa b)-I_{\mu}(\imath \kappa b) K_{\mu}(\imath \kappa r)\right]-\right. \\
& \left.-\frac{K_{\mu}\left(-\imath \kappa r^{\prime}\right)}{K_{\mu}(-\imath \kappa b)}\left[I_{\mu}(-\imath \kappa r) K_{\mu}(-\imath \kappa b)-I_{\mu}(-\imath \kappa b) K_{\mu}(-\imath \kappa r)\right]\right\} \kappa d \kappa d \mu .
\end{aligned}
$$

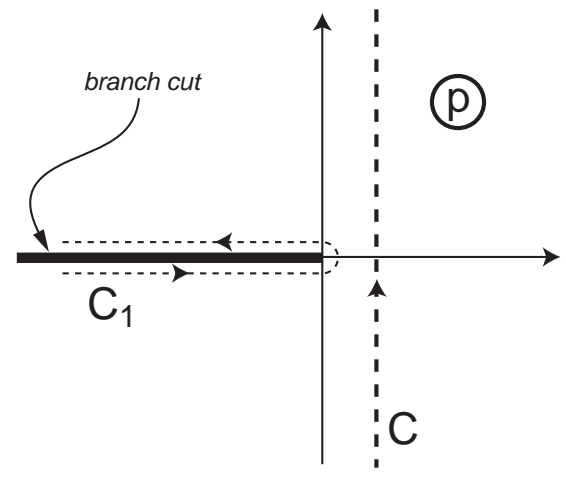

FIG. 4: Integration contours on the complex $p$-plane. Explanations are in the text.

The expression in curly brackets here can be simplified using the following three relations:

$$
\begin{aligned}
& I_{\mu}(\imath \kappa r) K_{\mu}(\imath \kappa b)-I_{\mu}(\imath \kappa b) K_{\mu}(\imath \kappa r)= \\
= & I_{\mu}(-\imath \kappa r) K_{\mu}(-\imath \kappa b)-I_{\mu}(-\imath \kappa b) K_{\mu}(-\imath \kappa r)= \\
= & -\frac{\pi}{2}\left[J_{\mu}(\kappa r) Y_{\mu}(\kappa b)-J_{\mu}(\kappa b) Y_{\mu}(\kappa r)\right], \quad
\end{aligned}
$$

and

$$
K_{\mu}(\imath \kappa b) K_{\mu}(-\imath \kappa b)=\left(\frac{\pi}{2}\right)^{2}\left[J_{\mu}^{2}(\kappa b)+Y_{\mu}^{2}(\kappa b)\right],
$$

and

$$
\begin{aligned}
& K_{\mu}\left(\imath \kappa r^{\prime}\right) K_{\mu}(-\imath \kappa b)-K_{\mu}\left(-\imath \kappa r^{\prime}\right) K_{\mu}(\imath \kappa b)= \\
= & -\frac{\pi^{2} \imath}{2}\left[J_{\mu}\left(\kappa r^{\prime}\right) Y_{\mu}(\kappa b)-J_{\mu}(\kappa b) Y_{\mu}\left(\kappa r^{\prime}\right)\right] \cdot(\mathrm{C} 10
\end{aligned}
$$

Using these three results, we directly see that the formula (C7) gets transformed into (9). This can, of course, be considered as another proof of normalization conditions for $Z$-functions, Eq. (B1B2).

\section{APPENDIX D: INTEGRATION OVER THE COORDINATE $r^{\prime}$ OF THE TRAJECTORY END}

Most easily, integration in (11) can be addressed using Eq. (5). Indeed, whatever is the value of $r$, we should consider the limit of large $t$ in the sense that $t a^{2} \gg r^{2}$; that means, by the time $t$ the walker should have traveled from its starting point typically much further than to the origin, $\mathcal{O}$. Then, we note that although the integration over $r^{\prime}$ runs to infinity, the integral is dominated by $r^{\prime}$ up to about $a \sqrt{t}$, because of the truncation by the exponential factor $e^{-r^{\prime 2} / a^{2} t}$. Accordingly, the argument $\xi=2 r r^{\prime} / a^{2} t$ of $I_{\mu}$ in the Eq. (5) is small, and we can use the expansion $I_{\mu}(\xi) \simeq(\xi / 2)^{\mu} / \Gamma(1+\mu)$. Upon integration over $r^{\prime}$, this yields

$$
\begin{aligned}
W(\theta) & \propto e^{-r^{2} / a^{2} t} \times \\
& \times \int_{0}^{\infty} \cos (\mu \theta) \frac{\Gamma\left(1+\frac{\mu}{2}\right)}{\Gamma(1+\mu)}\left(\frac{r^{2}}{a^{2} t}\right)^{\frac{\mu}{2}} d \mu .
\end{aligned}
$$

Now, we have to remember that $r^{2} / a^{2} t \ll 1$, which means that the latter integral is dominated by small $\mu$, more specifically by $\mu$ up to about $1 /\left|\ln \left(r^{2} / a^{2} t\right)\right|$. Replacing both $\Gamma$-functions with unity leads then to

$$
\begin{aligned}
W(\theta) & \propto \frac{\frac{1}{2} \ln \left(\frac{a^{2} t}{r^{2}}\right)}{\left(\frac{1}{2} \ln \left(\frac{a^{2} t}{r^{2}}\right)\right)^{2}+\theta^{2}} \simeq \\
& \simeq \frac{\frac{1}{2} \ln t}{\left(\frac{1}{2} \ln t\right)^{2}+\theta^{2}},
\end{aligned}
$$

where the latter transformation is justified again because $t$ is large. Thus, the resulting distribution is indeed independent of $r$, and it is nothing else but the Spitzer law, Eq. (11).

\section{APPENDIX E: PROOF THAT $W$ IS THE PROBABILITY}

Here, we check that $W$ satisfies the normalization condition as the probability:

$$
\sum_{n=-\infty}^{\infty} W\left(\theta+2 \pi n, \frac{3 r r^{\prime}}{a^{2} t}\right)=1,
$$

which also means that identification of the points $\theta$ and $\theta+2 \pi n$ erases all the topological information. 
First, let us denote for brevity $z=2 r r^{\prime} / a^{2} t$, and then we write

$$
\begin{aligned}
& \sum_{n=-\infty}^{\infty} W(\theta+2 \pi n, z)= \\
= & 2 e^{-z \cos \theta} \int_{0}^{\infty}\left[\sum_{n=-\infty}^{\infty} \cos ((\theta+2 \pi n) \mu)\right] I_{\mu}(z) d \mu= \\
= & e^{-z \cos \theta} \int_{-\infty}^{\infty}\left[\sum_{n=-\infty}^{\infty} \cos ((\theta+2 \pi n) \mu)\right] I_{|\mu|}(z) d \mu(\mathrm{E} 2)
\end{aligned}
$$

Here, integration is expanded over all $\mu$, both positive and negative, the price being the absolute value of $\mu$ serving as an index of $I_{|\mu|}$. The expression in the square brackets can be easily transformed using the identity

$$
\sum_{k=-\infty}^{\infty} e^{2 \pi \imath k t}=\sum_{m=-\infty}^{\infty} \delta(t-m)
$$

Thus, we write

$$
\begin{aligned}
& \sum_{n=-\infty}^{\infty} \cos ((\theta+2 \pi n) \mu)= \\
= & \frac{1}{2} \sum_{n=-\infty}^{\infty}\left[e^{\imath \mu(\theta+2 \pi n)}+e^{\imath \mu(-\theta-2 \pi n)}\right]= \\
= & \frac{1}{2} \sum_{n=-\infty}^{\infty}\left[e^{\imath \mu(\theta+2 \pi n)}+e^{\imath \mu(-\theta+2 \pi n)}\right]= \\
= & \frac{1}{2} \sum_{n=-\infty}^{\infty} e^{2 \pi \imath n \mu}\left[e^{\imath \mu \theta}+e^{-\imath \mu \theta}\right]= \\
= & \cos (\mu \theta) \sum_{n=-\infty}^{\infty} e^{2 \pi \imath n \mu}=
\end{aligned}
$$

$$
=\cos (\mu \theta) \sum_{m=-\infty}^{\infty} \delta(\mu-m)
$$

We also use the two identities $\underline{34}]$ :

$$
I_{\nu}=e^{-\imath \nu \pi / 2} J_{\nu}(\imath z)
$$

(formula 8.406.3 in [34]) and

$$
e^{\imath z \cos \phi}=J_{0}(z)+2 \sum_{k=1}^{\infty} \imath^{k} J_{k}(z) \cos (k \phi)
$$

(formula 8.511.4 in [34]). This yields

$$
\begin{aligned}
& \sum_{n=-\infty}^{\infty} W(\theta+2 \pi n, z)= \\
= & e^{-z \cos \theta} \sum_{m=-\infty}^{\infty} e^{\imath m \theta} I_{|m|}(z)= \\
= & e^{-z \cos \theta \sum_{m=-\infty}^{\infty} e^{\imath m \theta-\imath|m| \pi / 2} J_{|m|}(\imath z)=} \\
= & {[\underbrace{J_{0}(\imath z)+2 \sum_{e^{\imath(\imath z) \cos (\theta-\pi)}}^{\infty} \cos (m \theta-m \pi) \imath^{m} J_{m}(\imath z)}_{m=1}] \times } \\
\times & e^{-z \cos \theta}=1 .
\end{aligned}
$$

This completes the proof.
[1] F. Spitzer, Trans. Amer. Math. Soc., 87, 187-197, 1958.

[2] P. Messulam, M. Yor, J. Lond. Math. Soc., 26, n. 2, 348, 1982.

[3] M. Fisher, V. Privman, S. Redner J. Phys. A: Math. \& Gen., 17, p. L569-L578, 1984

[4] J. Pitman, M. Yor, The Annals of Probability, 11, n. 3, p. $733-779,1986$.

[5] J. Rudnick, Y. Hu, J. Phys. A: Math. \& Gen., 20, 44214438, 1987

[6] J. Rudnick, Y. Hu, Phys. Rev. Lett., 60, n. 8, 712-715, 1988

[7] B. Duplantier, H. Saleur, Phys. Rev. Lett., 60, n. 23, 2343-2346, 1988.

[8] J. Pitman, M. Yor, The Annals of Probability, 17, n. 3, p. 965-1011, 1989.

[9] C. Belisle, The Annals of Probability, 17, n. 4, p. 13771402, 1989.

[10] A. Comtet, J. Desbois, C. Monthus, J. Stat. Phys., 73, n. 1/2, 433-440, 1993.

[11] B. Drossel and M. Kardar, Phys. Rev. E, 53, 5861, 1996;
In: Theoretical amd Mathematical Models in Polymer Research, A. Grosberg, Editor, Academic Press, 1998, p. 187-217.

[12] K. Samokhin J. Phys. A: Math. Gen. 31 n. 44, p. 87898795, 1998.

[13] K. Samokhin J. Phys. A: Math. Gen. 31 n. 47, p. 94559468, 1998.

[14] S. F. Edwards, Proc. Phys. Soc. London, 91, 513, 1967

[15] S. Prager, H. L. Frisch, J. Chem. Phys., 46, n. 4, 14751483, 1967.

[16] F. W. Wiegel, in: Phase Transitions and Critical Phenomena, C. Domb and J.L. Lebowitz, eds, Academic Press, 1983, vol. 7, p. 101-149.

[17] P.-G. de Gennes Simple Views on Condensed Matter, World Scientific, Singapore, 1992.

[18] M. Doi, S. F. Edwards The Theory of Polymer Dynamics, Oxford University Press, Oxford 1986.

[19] V. Rybenkov, N. Cozzarelli, A. Vologodskii Proc. Nat. Ac. Sci., v. 90, 5307, 1993.

[20] S. Shaw, J. Wang Science, v. 260, 533, 1993. 
[21] V. Rybenkov, C. Ullsperger, A. Vologodskii, N. Cozzarelli Science, 277, 690-693, 1997.

[22] A. Yu. Grosberg Physics - Uspekhi, v. 40, 125-158, 1997.

[23] A. Yu. Grosberg Physical Review Letters, v. 85, 38583861, 2000.

[24] A. Yu. Grosberg preprint cond-mat/0207427

[25] A. Vologodskii, A. Lukashin, M. Frank-Kamenetskii Sov. Phys. JETP, v. 67, p. 1875, 1974; A. Vologodskii, M. Frank-Kamenetskii Sov. Phys. Uspekhi, v. 134, p. 641, 1981; A. Vologodskii Topology and Physics of Circular DNA, CRC Press, Boca Raton, 1992.

[26] K. Koniaris, M. Muthukumar Phys. Rev. Lett., v. 66, p. 2211-2214, 1991.

[27] T. Deguchi, K. Tsurusaki Phys. Rev. E, v. 55, n. 5, p. 6245-6248, 1997.

[28] D. W. Sumners, S. G. Whittington Journal of Physics A: Math.\& Gen., v. 21, p. 1689, 1988

[29] N. Pippenger Disc. Appl. Math., v. 25, 273, 1989.

[30] J.-L. Sikorav, B. Duplantier, G. Jannink \& Y. Timsit J. Mol. Biol., v. 284, p. 1279-1287, 1998

[31] S. Nechaev Statistics of knots and entangled random walks, (World Scientific: Singapore, 1996)

[32] A. Gray, G. B. Mathews A Treatise on Bessel Functions and Their Applications to Physics, McMillan, London, 1922 (pp. 66-69).

[33] S. Wolfram Mathematica: A system for Doing Mathematics by Computer, Addison Wesley, 1991.

[34] I. S. Gradshteyn, and I. M. Ryzhik Table of Integrals,
Series, and Products, A. Jeffrey (editor), Academic Press, 2000.

[35] R. Metzler, A. Hanke, P. Dommersnes, Y. Kantor, M. Kardar Phys. Rev. Lett., 88, 188101, 2002; R. Metzler, A. Hanke, P. Dommersnes, Y. Kantor, M. Kardar Phys. Rev. $E$, 65, 061103, 2002; O. Farago, Y. Kantor, M. Kardar cond-mat/0205111 R. Metzler, Y. Kantor, M. Kardar cond-mat/0206057

[36] J.-C. Meiners, S. Quake Phys. Rev. Lett., 84, 5014, 2000.

[37] P. Morse, H. Feshbach Methods of Theoretical Physics, NY, McGrow Hill, 1953 (Chapter 5.3).

[38] A.N. Semenov Zh. Eksp. Teor. Fiz., 1985, v. 88, p.1242.

[39] A. Yu. Grosberg, A. Feigel, Y. Rabin Physical Review E, v. 54, n. 6, p. 6618-6622, 1996.

[40] P.G. de Gennes Scaling Concepts in Polymer Physics, Cornell Univ. Press, Ithaca, NY, 1979.

[41] Using the notion of statistical weight, one has to be conscious of the issue of distinguishability of monomers. For instance, if all of them are exactly identical, then extra factor $t$ must be incorporated in the statistical weight of a ring. Here, we assume that since one monomer is anchored, the monomers are distinguishable by their numbering along the chain starting from the anchored place. It is also not difficult to establish that difficulties do not arise later, in Section VB when we consider a ring with no anchored monomer. We are indebted to I. Ya. Erukhimovich for this important comment. 